\title{
A Low Profile Electro-magnetic Actuator Design and Model for an Origami Parallel Platform
}

\author{
Marco Salerno, first author ${ }^{1}$ \\ EPFL \\ EPFL STI IGM RRL Station 9 CH-1015 Lausanne \\ e-mail: marco.salerno@epfl.ch \\ Amir Firouzeh, second author \\ EPFL \\ EPFL STI IGM RRL Station 9 CH-1015 Lausanne \\ e-mail: amirfirouzeh@epfl.ch \\ Jamie Paik, third author \\ EPFL \\ EPFL STI IGM RRL Station 9 CH-1015 Lausanne \\ e-mail: jamie.paik@epfl.ch
}

\begin{abstract}
Thin foldable origami mechanisms allow reconfiguration of complex structures with large volumetric change, versatility and at low cost; however, there is rarely a systematic way to make them autonomously actuated due to the lack of low-profile actuators. Actuation should satisfy the design requirements of, wide actuation range, high actuation speed and back-drivability. This paper presents a novel approach towards fast and controllable folding mechanisms by embedding an electromagnetic actuation system into a nominally flat platform. The design, fabrication and modeling of the electromagnetic actuation system are reported and a $1.7 \mathrm{~mm}$-thick single degree-of-freedom (DoF) foldable parallel structure reaching an elevation of $13 \mathrm{~mm}$ is used as a proof-of-concept for the proposed methodology. We also report on the extensive test results that validate the mechanical model in terms of the loaded and unloaded speed, the blocked force and the range of actuation.
\end{abstract}

\footnotetext{
${ }^{1}$ Corresponding author.
} 


\section{INTRODUCTION}

Major advances in robotic technology are leading to complex robot tasks in more challenging and random environments. We are now in a position to demand the presence of robots in daily life to improve our quality of life. Bringing traditionally powerful and fast but, large and unsafe robotic systems into daily life presents numerous engineering challenges, principally in the mechanical and geometric scalability of traditional mechanisms and actuators. In particular, portable robotic manipulators and highly interactive human-robot interfaces (HRI) require extremely demanding engineering specifications that call for novel solutions in design mechanisms and actuation systems. The structural foundations for many of these robotic devices are manipulators and grippers with numerous linkages and joints. The art of origami inspired robot designs whereby folding techniques give structural stability to thin lightweight composite materials allows transformation between quasi-2D to 3D configurations. Based on origami design we envision foldable pocket-size and millimeter-thick structures that can transform into robust 3D articulated mechanisms able to interact with the user by providing motion and/or force in a desired direction.

Smart composite microstructure (SCM) technology has emerged as a highperforming low cost innovative technique [1] for manufacturing thin composites that embed several functional features such as electrical connections, actuators and sensors. Researchers demonstrated the potential of this novel manufacturing technique by realizing different origami inspired robots capable of performing diverse tasks, including 
shape transformation [2], crawling locomotion [3-5], jumping [6] and multimodal locomotion [7]. At smaller scales SCM has also been used to develop millimeter-scale flying robotic insects [8]. Here we propose a mechanically simple yet effective and scalable design for HRI's in already existing platforms. We propose a nominally 2D miniature parallel system with embedded actuation and mechanical links for motion transmission that can evolve into a miniature haptic interface.

To make the system autonomous and reduce its size the actuation system should be one of the functional layers in the SCM. Embeddable actuators have to be thin, contained in the millimeter size of the system and have a wide range of speed (1-100 $\mathrm{mm} / \mathrm{s}$ ) and force (at least $200 \mathrm{mN}$ ) for interacting with human hands. Due to the system's target size and required capabilities it is hard to use current commercial actuators and components. Given the need for fast and repeatable motions in HRI's, thermally-activated actuation methods, whether for permanent shape transformation [2] or repeatable motion using shape memory alloy (SMA) actuators [4-7, 9-12], can be ruled out. An alternative to SMAs, piezoelectric actuators [13] provide higher speeds (10 to $50 \mathrm{~mm} / \mathrm{s}$ ) but due to their limited stroke, require the design of a complex transmission for employment. Aiming to achieve an actuation system with a wide stroke, high speed and low-profile, we chose electromagnetic actuation because of ease of manufacturing planar coils by photolithography process as well as the possibility of embedding this actuation into a quasi-2D structure using the SCM technique.

Several studies describe the design and fabrication of coupled magnets and coils at the meso and microscale [14]. Miniature coils are used in wireless power 
transmission [15-17], tactile sensors [18] and medical imaging [19]. For actuation purposes, millimeter or sub-millimeter scale motion has been obtained by exploiting, in many cases, the simple axial repulsion of a planar coil and an axially magnetized cylindrical magnet [20-24].

In this paper we present the design, model and working prototype of a millimeterthick linear electromagnetic actuator that can be embedded into a robotic nominally flat platform. This design allows us to control the magnetic field to modulate actuation up to $13 \mathrm{~mm}$ from a $1.7 \mathrm{~mm}$ platform. We demonstrate the functionalities and performance with a one-degree of-freedom (DoF) origami parallel platform that can be reversibly folded from a planar configuration to a tri-dimensional pop-up structure.

The major contributions of this work are:

- The design, model and performance characterization of a novel nominally 2-D magnetic actuation system based on planar coils.

- The design and fabrication of a quasi-2D parallel platform using SCM fabrication that embeds multiple essential components such as folding linkages and the actuation system into the different layers of the structure.

- A quasi-2D parallel origami platform prototype that can repeatedly reconfigure between a planar state and a 3D pop-up configuration.

- The experimental validation of the model and prototype and a comprehensive study of the prominent physical principles affecting the behavior. 


\section{SYSTEM OVERVIEW}

The proposed planar to pop-up mechanism for a portable HRI systems is compact; its construction by SMC fabrication process makes the fabrication accurate, robust and rather cheap. Figure 1 illustrates the overview of the pop-up platform mechanism, a 1.7$\mathrm{mm}$ thick, pocket-sized pop-up foldable device, used as a proof-of-concept for the novel actuation method design and manufacturing process.

The actuators, placed at the extremities of the platform are composed of two planar layered coils, a rail structure and an axially magnetized permanent magnet. The coils are designed to make the magnet slide along the rail when they are energized thus obtaining a linear motion [25]. Each leg in this platform constrains four out of the 6- DoF of the centerpiece. As the constraints between each leg are coupled, the overall mechanism consisting of three legs has 1 active DoF. In Figure 2 we show a schematic of the actuator components and the actuation principle. In Figure 3, we present an overview of the layers composing the platform. The design, fabrication, modeling and testing of the platform parts components are presented as follows: In Section III.A we present the kinematics of the platform's pop-up mechanism, in Section III.B we discuss the model of the actuation system and its design. Then we depict the fabrication steps of the layered coils (Section IV.A) and the actuation mechanism (Section IV.B) of the platform (Section IV.C). In Section V.A we introduce the formula describing the different physical principles for predicting platform performance, such as Joule-heating and inductors' dynamics; in Section V.B we present the actuator model while in Section V.C 
the dynamic model of the pop-up platform is visited. In Section VI, we illustrate the experiments conducted on the coils to verify their dynamics and on the actuator for determining its dynamic constants including the friction coefficients; these parameters are then used in Section VII where the results of the pop-up platform motion prediction are shown. In Section VIII we conclude by discussing present achievements and future developments.

\section{DESIGN PARAMETER SELECTION}

The performance of the prototype pop-up platform is determined by the geometrical parameters of the mechanism, which dictate the transmission ratio between the input and output strokes and velocities, as well as by the effectiveness of different configurations. Below we present the design parameters for the platform based on these considerations.

\section{A. Mechanism kinematics}

To obtain the kinematics of the parallel mechanism shown in Figure 1, we modeled the resulting mechanism of each leg as equivalent to the double slider mechanism presented in Figure 4. In this configuration, the horizontal slider, link 1, contains the permanent magnet and the motion is controlled by the magnetic field generated by the coils. Link 1 has a single DoF dictated by the rail into which it moves. The mechanism transforms the in-plane motion of link 1 to the vertical motion of the second slider (link 3) through the interaction with link 2. 
The transmission ratio between the input link, link 1 , and the output link, link 3 , is determined by the length of the link 2 and the position of link 1 in the rail according to the following equations, (1) and (2):

$$
\begin{aligned}
& y_{3}=\sqrt{l^{2}-\left(l-x_{1}\right)^{2}} \\
& \dot{y}_{3}=\frac{\dot{x_{1}}\left(l-x_{1}\right)}{\sqrt{l^{2}-\left(l-x_{1}\right)^{2}}}=\frac{\dot{x_{1}}}{\tan \theta_{2}}
\end{aligned}
$$

The equation for the velocity (Eq. 2) of the links suggests a nonlinear transmission between link 1 and link 3 velocities. In the flat state, the mechanism is at a singular point and as it starts moving upwards, the ratio of the output speed to the input speed decreases according to (2). In future, this transmission ratio can be exploited further to obtain a desired force profile on the output link. Decreasing the velocity ratio, the mechanism has a higher force transmission at larger angles that is desirable since it can overcome increasing forces as it moves toward its final position. The current pop-up platform is over-constrained with the third slider. Despite the over-constraint, this design has two advantages; 1 ) it reduces the forces transmitted to the rails (since the inplane force components of the three legs exerted on the centerpiece cancel each other out) which reduces significantly the friction forces; 2 ) the output vertical force from the centerpiece is increased by increasing the number of actuation points. Also, the overconstraint mechanism issues are alleviated via clearance between the leg and the rail. The dynamic modeling of this structure, which is necessary to understand the forces acting on the mechanism and to optimize future designs, is discussed in Section V.C. 


\section{B. Actuator design}

The proposed actuator, as described in Figure 5, is composed of two planar coils, a rail structure and an axially magnetized permanent magnet. We designed the coils to make the magnet slide along the rail when they are energized thus obtaining a linear motion. The advantage of this strategy is that the magnetic field is managed along the rail to allow maximum of $8 \mathrm{~mm}$ stroke, but the transmission will redirect the stroke in the vertical direction thus obtaining $13 \mathrm{~mm}$ actuation strokes of a $1.7 \mathrm{~mm}$ structure. The magnet movement is initiated by the combination of repulsive force from the first coil and attractive force from the second one. The magnetic fields of the two planar coils interact and generate the electromagnetic force on the permanent magnet.

Although the magnetic forces applied to a permanent magnet drop by a power of 3 (since it is dependent on magnet volume) when the magnetic system is scaled down, the force to weight ratio does not suffer from this reduction. Furthermore, with the same current density it is possible to reach higher actuation velocities. While decimeter scale coils use cylindrical wires, miniature planar coils use micron-thick flat conductors with rectangular cross section; this further increases the conductor surface/volume ratio, boosting the thermal exchanges and as a consequence allowing higher current densities compared to the decimeter scale where thermal exchanges are not very efficient; this, upon a scaling of $1 / k$, increases the magnetic interaction of a factor $k$ as explained by [26] and [27]. It is difficult to obtain wide motions at millimeter scale by exploiting the axial repulsion of a planar coil and an axially magnetized cylindrical magnet. This is challenging [20-24] due to the drop in magnetic forces with the increase in permanent 
magnet and coil inter-distance. For this reason, the use of a linear electromagnetic actuator combined with proper transmission seems ideal for a better coil-magnet distance management.

The permanent magnet used in the prototype is a $1 \mathrm{~mm}$-long cylinder with $10 \mathrm{~mm}$ diameter. It is axially magnetized and has a magnetic flux remanence of $1.2 \mathrm{~T}$ (HKCM, Germany). The coils are designed with slightly larger diameter, $12 \mathrm{~mm}$, the stroke will be smaller than the diameter of the coil since it is expected that, when the magnet is lying in the center of one of the two coils, the repulsive or attractive forces would be normal to the direction of the rail and would not result in action of the mechanism. For these reasons we chose $8 \mathrm{~mm}$ to be conservative and ensure magnet motion.

In order to define the other system design parameters, finite elements analysis (FEA) to evaluate the forces between the coils and the magnet was executed using the AC/DC module of Comsol 5.2. The forces applied by the coils to the magnet have been considered, as first approximation, magnetostatic. For this purpose, a stationary study was set using the following equations:

The ampere Law for static cases defined as

$$
\nabla \times H-\sigma v \times B=J_{e}
$$

The definition of magnetic potential is

$$
\mathrm{B}=\nabla \times \mathrm{A}
$$

The constitutive relation for the magnet is

$$
\mathrm{B}=\mu_{0} \mu_{r} H+B_{r}
$$


The constitutive relation for the coils is

$$
\mathrm{B}=\mu_{0} \mu_{r} H
$$

And the externally generated currents for the coils are

$$
J_{e}=\frac{N I_{\text {coil }}}{a_{s}} e_{\text {coil }}
$$

Where Je (7) is an externally generated current density, $v$ is the velocity of the conductor, $\sigma$ the electrical conductivity, $H$ the magnetic field (3), $A$ the magnetic potential (4), $B$ is the flux density (6), $B r$ the residual flux density (5), $\mu_{0}$ the magnetic permeability in vacuum, $\mu_{\mathrm{r}}$ the relative permeability of the material, $N$ the number of turns of the coil, $I_{\text {coil }}$ the current energizing the coil, as is the cross sectional area of the coil domain and $e_{\text {coil }}$ is the coil current flow [28]. The coil height was set at $0.5 \mathrm{~mm}$ to be embedded into a millimeter thick structure. The magnetic forces are calculated using Maxwell's stress tensor; in particular, a numeric integration on the surface of the object that the force acts on, was performed. The aspect ratio of the magnets and coils was around 0.05 , making computationally heavy meshing and solving the model, but we obtained a stable solution by using a gradient in mesh nodes distribution and increasing element number at around 106.

We studied the magnet-coils interaction forces for different positions along the direction of motion and at different elevations; we used this simulations set to evaluate the magnetostatic forces acting on the magnet. We also analyzed the interaction forces between two magnets at different distances; we used this simulation set to evaluate the minimum distance at which two coplanar magnets generate negligible interaction 
forces. We used this result to design the platform with three legs. Regarding the magnet-coils interactions, we performed a parametric study varying magnet position in $\mathrm{x}$ and $\mathrm{y}$ directions according to the reference system reported in Figure 6 . In the simulation, we modified iteratively the current and conductor turns along with the maximum number of coil turns that was possible to fabricate with the used technique. The constants used in the model are reported in Table I. We used simulation results to estimate the ampere/turns needed to perform motion thus getting an indication of the number of turns and layers for the planar coils design. The magnetostatic forces calculated with the simulations were used for the system motion prediction presented in Section V.B.

We conducted a parametric study considering the magnet position along the $z$ axis; this was useful as magnet elevation depends on the platform design; the simulation results are reported in Figure 6. In the prototype described in Section IV.C, the coils-magnet distance in the $z$ direction is $1.4 \mathrm{~mm}$. Regarding the magnet-magnet interaction; we performed a parametric study of two coplanar magnets, varying their distance. According to the simulations, at a distance of $45 \mathrm{~mm}$ the interaction forces between the magnets were lower than $0.1 \mathrm{mN}$, three orders of magnitude lower than the maximum force applied by the coil system $(50 \mathrm{mN})$; therefore, they can be considered negligible. We then considered this distance limit in the design of the pop-up parallel platform in order to avoid magnetic interactions between the legs. Table II and Figure 7 reports the mechanism's sizes and values. 


\section{MANUFACTURING}

Accurate, robust and repeatable manufacturing technique is imperative to keep the overall dimension of the platform at a minimum, while maximizing the output force and the range of motion. In the following subchapters we will explain the fabrication of the coils and articulated mechanism and their integration into the millimeter thick actuated parallel platform.

\section{A. Printed coils}

We manufactured the coils using the photo-engraving technique on Pyralux flexible thin sheets of $32 \mu \mathrm{m}$ thickness with a conductor thickness of $12 \mu \mathrm{m}$. We inverted the winding sense of the stacked coils in order to sum the magnetic field produced by the single coils as proposed by [15]. To electrically connect overlapping coil layers in series, we first used a UV laser micromachining system (DCH-355-4 laser head from Photonics Industries Inc.) [29] to remove the Kapton layer from the back of the coil, exposing the conductor and then applied conductive epoxy glue (Chemtronics CW2460) to electrically bond six adjacent layers.

One of the goals of this work is to minimize the overall thickness of the platform while targeting high actuation speeds; the initial criteria for maximizing the produced magnetic field was maximizing the number of turns in the single layer coil without stacking too many layers, thus, we tried to minimize the conductive path width avoiding short circuits and open circuits. The photoresist has a $15 \mu \mathrm{m}$ resolution; therefore, coils 
with a path width of $50 \mu \mathrm{m}$ and a distance between two turns of $100 \mu \mathrm{m}$, were investigated.

First, we defined the coil shape in Matlab (MathWorks, US) as a sequence of coordinates and elaborated it with Altium designer (Altium, US) for printing the mask used for the circuit impression. The parameters such as the UV exposition time, the photoresist development time and the etching time were iteratively changed in order to increase the successful rate of the coils. Second, in order to compensate for the underetching, we tested masks with different path thicknesses and the results were compared. For example some of the masks used and resulting coils are shown in Figure 8. The criteria for choosing the fabrication parameters were 1) the low deviation of electrical resistance experimental values with theoretical ones 2) a low percentage of failures, considering as failed a coil with broken conductors or with resistance deviating $20 \%$ or more from the theoretical values. We estimated the resistance of the coil according to the formula $R=\rho^{*} L / A$, where $\rho$ is copper resistivity $(1.68$ e- $8 \Omega / \mathrm{m}), L$ is the length of the conductor of the planar coil (calculated as $1.122 \mathrm{~m}$ for the used coils of 12 $\mathrm{mm}$ diameter and having 55 turns), and $A$ is the section of the conductor corresponding to a width of $50-80 \mu \mathrm{m}$. A mask having $70 \mu \mathrm{m}$ for the conductive path and $30 \mu \mathrm{m}$ for the insulating path gave the most robust results $(20.9 \pm 3.2 \Omega$ where the theoretical value was $22.5 \Omega$ ) and was used for coil manufacturing. 


\section{B. Structure and mechanism}

The dimensions of the mechanism were designed to have negligible interaction of the magnetic field in each two legs. The design was based on simulations that used the predetermined design parameters (Section II.B): the magnet size (10 $\mathrm{mm}$ in diameter), the magnet in rail motion range $(8-10 \mathrm{~mm})$ and the diameter of the coils $(12 \mathrm{~mm})$. The minimum distance $d$ (distance of the magnets at the pop-up state) of $45 \mathrm{~mm}$ was required. This initial design, used to study the behavior of the system, follows the criteria of low weight and avoidance of magnet-magnet interactions. The optimized design solutions in agreement with other criteria will be investigated in future work.

The distance between the center of the platform and center of the magnet during motion, $\mathrm{a}_{\mathrm{min}}$, resulted around $20 \mathrm{~mm}$, being $\mathrm{d} 45 \mathrm{~mm}$ (result from the simulations in Section II.B); since the actuation range was $8 \mathrm{~mm}$, the length $a_{\max }$ in mechanism extended configuration was $28 \mathrm{~mm}$. The minimum length $\mathrm{b}$, of the structure embedding the magnet was $12 \mathrm{~mm}$ (considering $1 \mathrm{~mm}$ of material around the magnet); we maintained the same size for, $r_{p}$, central structure size $(6 \mathrm{~mm})$. Consequently, we assigned a value of $c=a_{\max }-r_{p}-b / 2,16 \mathrm{~mm}$ to the length $c$ of the articulated link. The vertical range of motion of the platform was expected to be $12 \mathrm{~mm}$.

C. Pop-up parallel platform prototype description

We fabricated the platform by integrating layers of different materials with specific functionalities to obtain a multifunctional composite. The prototype is composed of the following functional layers (Figure 3): 
- the bottom layer that provides the anchoring plane for the platform and protects sensitive coil layers;

- the coil layers that provide the required magnetic field;

- two, patterned glass fiber layers with a Kapton layer embedded between them that constitute the articulated mechanism;

- four glass fiber layers that cover the coil system and form the rails for the sliders of the mechanism.

Figure 3 shows the schematic of the layers, Figure 9 shows a picture of the final prototype and Figure 10 reports the platform size and weight. Table III reassumes the platform element's mass. We machined the desired patterns in different layers including the structural $0.2 \mathrm{~mm}$ glass fiber layers, the $32 \mu \mathrm{m}$ coil layers and the $25 \mu \mathrm{m}$ Kapton layers. We aligned and piled up these layers using the reference holes and a jig. We connected the layers with thermo-adhesive inter-layers using a heat-press. The final prototype, shown in Figure 9, has a thickness of $1.7 \mathrm{~mm}$.

\section{MODELLING}

To identify the predominant physical effects influencing mechanism motion, we sequentially analyzed and modeled the coil system, the actuator, and the pop-up parallel platform. We estimated and experimentally verified the coils' time response, the temperature change and the friction between the slider and the rail. 


\section{A. Actuation system model}

The pop-up parallel system is powered through multiple planar coil stacks. In this section, we report the estimation of the coil system time response; we report its experimental verification in Section VI.A. For verifying the time constant of the stacked coil system, we calculated the coils' inductance and experimentally verified the corresponding value.

As the first approximation, the coil was assumed a series of loops with the same diameter; the theoretical formula [30] that we used for estimating the inductance $L_{\text {circle }}$ is:

$$
\mathrm{L}_{\text {circle }} \approx \mathrm{N}^{2} \mathrm{R} \mu_{0} \mu_{\mathrm{r}}\left[\ln \frac{8 \mathrm{R}}{\mathrm{a}_{1}}-2\right]
$$

Where $\mathrm{N}$ is the number of turns, $\mathrm{R}$ is the radius of the circle, al is the wire radius, $\mu_{0}$ and $\mu_{\mathrm{r}}$ are the magnetic permeability in vacuum $\left(\mu_{0}=1.2567 \mathrm{H} / \mathrm{m}\right)$ and the relative permeability of the medium, in this case, the air $\left(\mu_{r}=1.00000037\right)$. An inductance of $1.4161 \mathrm{mH}$ resulted from a series of six coils having 300 turns, $50 \mu \mathrm{m}$ of conductor width and an average radius of $6 \mathrm{~mm}$. We considered a resistance of $21 \Omega$ for each layer, as found in Section IV.A). The resulting time constant, $\tau$, was $11.2 \mu \mathrm{s}$. Since we evaluated from the experiments that actuation times were around $0.1 \mathrm{~s}$ we neglected electromagnetic transient effects due to magnetic field generation. Transient thermal response is another aspect that can change the magnetic field by affecting the electrical resistance of the coils. For describing this effect, we used the linear correlation [31] between the temperature increase and the resistance increase:

$$
\mathrm{R}=\mathrm{R}_{0}\left[1+\propto\left(\mathrm{T}-\mathrm{T}_{0}\right)\right]
$$


Where the electrical resistance, $R$, at the temperature, $T$, depends on the resistance, $R_{0}$, at the temperature, $T_{0}$, and on the temperature coefficient, $\alpha$. Further, considering the power consumption after the magnetic field reaches the steady state, the power, $P$, converted to electrical energy due to the current, $I$, flowing in the resistance, results in:

$$
\mathrm{P}=\frac{\mathrm{V}^{2}}{\mathrm{R}}
$$

While the temperature increase, $d T$, due to the heat, $d Q$, supplied to the conductor is:

$$
\mathrm{dQ}=\mathrm{Pdt}=\left(\mathrm{m}_{\mathrm{c}} \mathrm{c}_{\mathrm{c}}+\mathrm{m}_{\mathrm{k}} \mathrm{c}_{\mathrm{k}}\right) \mathrm{dT}
$$

Where $m_{c}$ and $m_{k}$ are the mass of the conductor and the mass of the Kapton in contact with the conductor, $c_{c}$ is the specific heat of the copper and $c_{k}$ is the specific heat of the Kapton. By multiplying (11) for the activation time $t$ and integrating (12), the following equation is obtained:

$$
\left(\mathrm{m}_{\mathrm{c}} \mathrm{c}_{\mathrm{c}}+\mathrm{m}_{\mathrm{k}} \mathrm{c}_{\mathrm{k}}\right)\left[\mathrm{T}^{2} \frac{\propto}{2}+\mathrm{T}\left(1-\propto \mathrm{T}_{0}\right)-\mathrm{T}_{0}+\propto \frac{\mathrm{T}_{0}^{2}}{2}\right]=\frac{\mathrm{V}^{2}}{\mathrm{R}_{0}} \mathrm{t}
$$

Solving equation 13 , the temperature $T$ of the coil after a time, $t$, was calculated, the values used for the constants are reported in Table IV. Thermal exchanges were not considered in this work since the heating rate of the coil was expected to be fast and the materials the coil is in contact with do not have a high thermal conductivity (Kapton and glass fiber). Although the proposed model is expected to overestimate the temperature increase of the coil, the trend of current decrease due to the temperature rise is expected to be similar to the experimental case. 


\section{B. Rail and slider model}

In this Section we examine the actuator, in particular the interaction of magnet, the couple of coils that are energized in the opposite sense, and the rail constraining magnet motion. We used this model to identify the parameters affecting the rail and slider interaction. These parameters were evaluated using the experiment on one actuator and they were then used to predict the pop-up platform behavior. The main forces acting on the magnet are the magnetic forces applied by the coils to the magnet considered dependent by their relative position and by the current flowing in the coils; the interaction of the magnet with the rail, modeled with static and dynamic friction where the force generating friction is the magnetic component perpendicular to the direction of motion and also the weight of the magnet; the constant force $C$ opposite to the direction of motion exerted by the bottom part of the rail on which the magnet slides.

The following equations describe actuator behavior:

$$
\begin{aligned}
& F_{m x}-F_{f r}-C=m \ddot{x} \\
& F_{f r}=\operatorname{sign}(\dot{x})\left(F_{m z}+m g\right) \mu_{n} \\
& F_{m x}=f(x, V) Q, \quad F_{m z}=f(x, V) Q
\end{aligned}
$$

Where $F m_{X}$ and $F m_{Z}$ are the magnetostatic forces in the direction of motion and in the direction perpendicular to coil plane. $F m_{X}$ and $F m_{Z}$ are functions of the magnet position $\mathrm{x}$, the powering voltage $V$ and the coil quality factor $Q$, ranging from 0 to 1 , that we use to consider the coils turn loss by leakages of the conductive glue during the multilayer bonding. The values of the magnetic forces were obtained by lookup tables 
over the FEM pre-calculated results shown in Section III.A. The constant $m$ is the mass of the magnet moving into the rail, $g$ the gravitational acceleration, $F_{f r}$ is the friction force and $\mu \mathrm{n}$ is the friction coefficient. To determine the unknown parameters $\left(\mu_{n}\right.$ and $\left.C\right)$ in (14) and (15) that model the interaction of the slider and the rail, we did a grid search to find the values that resulted in the best fit of the simulation results. (14) and (15) are solved numerically in a Simulink model and by the experimental results that will be presented in Section VI.B. We used these parameters for estimating the behavior of the whole mechanism.

\section{Pop-up platform}

Up to this point the model for the actuation system and the interaction between the slider and the rail have been presented. To model the behavior of the platform and predict its output force and displacement we integrated the models for the actuation system and the slider in an overall dynamical model of the platform. We verified the proposed model for the pop-up platform by experimental comparison, as reported in the next section. Due to the symmetry of the structure, the kinematics of the pop-up platform is similar to that of a double slider and the force that the platform can generate on the middle part is the sum of the vertical force that three such double slider mechanisms generate. The kinematics of the mechanism is dictated by the angle $\theta_{2}$. These equations can be rearranged to present all the variables as the input motion of the link 1, but the following representation is more convenient in the dynamic analysis of the mechanism. 


$$
\begin{gathered}
\mathrm{x}_{1}=\mathrm{l}\left(1-\cos \theta_{2}\right), \dot{\mathrm{x}_{1}}=1 \dot{\theta_{2}} \sin \theta_{2}, \ddot{\mathrm{x}_{1}} \\
=\mathrm{l}\left(\ddot{\theta_{2}} \sin \theta_{2}+\dot{\theta_{2}}{ }^{2} \cos \theta_{2}\right) \\
\mathrm{z}_{3}=1 \sin \theta, \dot{\mathrm{z}_{3}}=1 \dot{\theta_{2}} \cos \theta_{2}, \ddot{\mathrm{z}_{3}} \\
=\mathrm{l}\left(\ddot{\theta_{2}} \cos \theta_{2}-\dot{\theta_{2}} \sin \theta_{2}\right) \\
\mathrm{x}_{2}=\mathrm{x}_{1 / 2}, \dot{\mathrm{x}_{2}}=\dot{\mathrm{x}_{1}} / 2, \ddot{\mathrm{x}_{2}}=\ddot{\mathrm{x}_{1}} / 2 \\
\mathrm{z}_{2}=\mathrm{z}_{3} / 2, \dot{\mathrm{z}_{2}}=\dot{\mathrm{z}_{3}} / 2, \ddot{\mathrm{z}_{2}}=\ddot{\mathrm{z}_{3} / 2}
\end{gathered}
$$

The free body diagrams of the 3 links are presented in Figure 11. The following are the governing dynamic equations for the mechanism.

$$
\begin{aligned}
& \mathrm{F}_{\mathrm{y}(3-2)}=\mathrm{m}_{3}\left(\mathrm{~g}+\ddot{\mathrm{y}_{3}}\right)+\mathrm{F}_{\text {Load cell }} \\
& \mathrm{F}_{\mathrm{y}(1-2)}=\mathrm{F}_{\mathrm{y}(3-2)}+\mathrm{m}_{2}\left(\mathrm{~g}+\ddot{\mathrm{y}_{2}}\right) \\
& \mathrm{F}_{\mathrm{x}(1-2)}=\mathrm{F}_{\mathrm{y}(1-2)} \cot \theta_{2}+1 / 2 \mathrm{~m}_{2} \cot \theta_{2} \ddot{\mathrm{y}_{2}} \\
& \quad+1 / 2 \mathrm{~m}_{2} \ddot{\mathrm{x}}_{2}+\frac{\mathrm{I}_{2} \ddot{\theta_{2}}}{\mathrm{l}_{2} \sin \theta_{2}}+2 \mathrm{k}_{\text {hinge }} \theta_{2} \\
& \mathrm{~F}_{\mathrm{y}(\text { rail })}=\mathrm{F}_{\mathrm{y}(1-2)}+\mathrm{m}_{2} \mathrm{~g}-\mathrm{F}_{\mathrm{mz}} \\
& \mathrm{m}_{1} \ddot{\mathrm{x}_{1}}=\mathrm{F}_{\mathrm{mx}}-\mathrm{F}_{\mathrm{fr}}-\mathrm{F}_{\mathrm{x}(1-2)} \\
& \mathrm{F}_{\mathrm{fr}}=\operatorname{sign}(\dot{\mathrm{x}})\left(\mathrm{F}_{\mathrm{y}(\text { rail })}\right) \mu_{\mathrm{n}}-\mathrm{C}
\end{aligned}
$$

In the fabricated prototype, the hinges have a width, $b$, of $10 \mathrm{~mm}$, a length, $L$, of 1 $\mathrm{mm}$ and a thickness, $h$, of $25 \mu \mathrm{m}$; the elastic module, $E$, of the Kapton layer is $2 \mathrm{GPa}$. We experimentally evaluated the hinge's rotational stiffness, $k_{\text {hinge, }}$ through repeated tests using small weights in different angular positions; it resulted to $0.84 \pm 0.3$ e- $6 \mathrm{Nm} / \mathrm{rad}$. Such stiffness can also be used to calculate overall structure stiffness [32] These 
equations (18-27) dictate the behavior of the mechanism. The estimations based on them were used to study the experimental results and to make sense of the trends in the results for various loading and actuation scenarios.

\section{EXPERIMENTS FOR VERIFING MODEL ASSUMPTIONS AND SYSTEM PARAMETERS CHARACTERIZATION}

The model presented in the Section $V$ enables us to predict and explain the behavior of the pop-up platform and to design more complex mechanisms based on the same actuation principle. The experiments reported in this Section were designed to verify the negligible effects, such as transient thermal and electromagnetic effects and to characterize the unknown parameters in the model ( $\mu_{n}, C$, and $\left.Q\right)$.

As the first set of experiments, we measured the magnetic field flux density, $B$, variation produced by the coils upon activation, as shown in Figure 12a. We used this test to verify the coil system time constant and the increase in resistance due to the temperature increase. As the second set of experiments, we measured the motion of a single magnet propelled by the coils, as shown in Figure $12 \mathrm{~b}$. This test set was used to evaluate the parameters affecting the rail and slider interaction, such as the friction coefficient, $\mu \mathrm{n}$, the external force, $C$, and the coil quality factor, $Q$.

\section{A. Coil system tests}

To verify the value of the time constant, $\tau$, obtained as reported in Section IV.A, we placed a Hall Effect sensor (SS490, Honeywell, USA) on the coil system and recorded the variation of magnetic field flux density, $B$, in time, during energization with input 
voltages of $20 \mathrm{~V}$ and $30 \mathrm{~V}$. We manually fixed the sensor over the coil, approximatively at the center of the external one, at a vertical distance of $1-2 \mathrm{~mm}$. Since this test was used to verify the time response of the system, the placement of the coil is not critical as long as the produced field is in the sensitivity range of the sensor.

Figure 13 shows that the experimentally evaluated time constant, $\tau$, was around 7 $\mu \mathrm{s}$, smaller than the theoretical value of $11.2 \mu \mathrm{s}$. This is due to the loss of some turns in the stacking process caused by the flow of excess conductive glue that reduced the ratio of the inductance to resistance. In future works, improved fabrication methods and using some of the proposed inductance calculation methods should make the experimental results closer to the theoretical ones.

We also measured the B field in time, to verify the increment in the electrical resistance due to the increase in temperature, as described in Section V.A, by maintaining the powering voltage for $0.2 \mathrm{~s}$ in order to appreciate the thermal effect, thus identifying at different voltages its influence on the produced magnetic field flux density. In order to compare the model result with the experiments, since, $B$, is proportional to the current flowing in the coil, I, the theoretical normalized current decrease in time was calculated. In the tested prototype we could not easily evaluate shortcomings in the actual prototype, for example the shortcuts in the coils' turns due to conductive glue leakages, conductive glue temperature effect and the boundary conditions for the thermal exchanges.

These factors contribute to slightly decrease the heating rate of the coil as Figure 14 shows, but the linear trend in the investigated time span confirmed the magnetic 
field decreases due to the increase in temperature. We report this in Figure 14 and this effect can be considered negligible in the timespan of the actuation (less than $0.1 \mathrm{~s}$ as shown in Section VII.A). The experimental results presented in this Section verified that the transient electromagnetic behavior was too fast and the transient thermal behavior was too slow compared to the actuation time span. So both effects are negligible and were not be included in the model of the platform.

\section{B. Actuator tests and determination of the dynamic parameters}

Friction coefficient, $\mu_{n}$, the external force, $C$, and the coil quality factor, $Q$, are three parameters in the model that are highly dependent on fabrication precision and quality; therefore, their values must be verified experimentally. Here we present the characterization of these parameters by studying the motion of a single slider as it is propelled by the coils and moves along the rail.

The slider motion is constrained by the rail structure as described in Section III.B. We applied input voltages to the coil system of 5 to $30 \mathrm{~V}$ with $5 \mathrm{~V}$ increments while recording the motion of the slider with a high-speed camera (FS700RH, Sony, Japan) at $960 \mathrm{fps}$. We repeated each test 3 times for each of the three rails of the platform. We report in Figure 15b the raw data for single repetition at the different voltages. At $5 \mathrm{~V}$ the magnet was repulsed by the first coil and attracted by the second, in agreement with the $F_{x}$ reported in Figure 15a. Since the momentum that is built up at this voltage was rather small, the magnet stopped before reaching the position with zero horizontal force. At $10 \mathrm{~V}$, the magnet built up enough momentum to surpass the zero force 
position but when it comes to a halt, the magnetic force was not large enough to pull it back to the zero force position. At higher voltages the magnet not only surpassed the zero force point but also went further, into the zone where the attraction force moved it back. Finally at $30 \mathrm{~V}$ it changed direction twice before stopping. The same motion was simulated using the model reported in Section V.B and the parameters were iteratively adjusted to replicate the behavior. These parameters are reported in Table V. For an easier comparison of simulation and experimental results, some features of the curve, stroke versus time, were evaluated in both simulations and experiments and were used in the comparison. In Figure $15 \mathrm{c}$ the characteristic maximum position, final position, maximum speed and motion time are highlighted. Experimental results that combine the different rails' outcomes were compared with the model results where the average of the $\mu_{r s}, \mu_{r d}$ and $C$ parameters is used. The results of the comparison are reported in Figure 16.

\section{POP-UP MECHANISM PERFORMANCE AND MODEL VERIFICATION}

In this Section, we report the study of the pop-up platform performance to demonstrate the effectiveness of the actuation method, the design and the manufacturing of the platform. By comparing the model's prediction of the behavior of the mechanism to the test results, we also confirmed the system model. In the first set of experiments, we measured the motion of the pop-up platform as Figure 17a shows. This test set was used to verify the dynamic model of the mechanism. In the second set, we loaded the pop-up platform with varying masses, as shown in Figure $17 \mathrm{~b}$. This test set was used to 
validate the dynamic model of the mechanism and the workspace reduction upon changes of the payload.

\section{A. Motion of the Pop-up platform}

We used the position tracking of the pop-up platform motion to demonstrate the performance of the system and to verify the presented actuator and mechanism model. The platform moves out of the plane upon the coils' activation, as described in Section II. Input voltages from $20 \mathrm{~V}$ to $50 \mathrm{~V}$, with $5 \mathrm{~V}$ increments, were applied to the coil system and we measured the top element position in time with the high-speed camera. We executed the recording from the side of the system and report the result of the tracking in Figure 18; platform elevation, speed and acceleration in time for different input voltages. Another test was performed to verify the dynamic model of the mechanism and the workspace reduction upon changes of the payload. We executed the same tracking by placing different sets of weights on top of the platform.

For each weight, we identified the minimum voltage to generate motion and the starting platform position; we measured the resulting position in time with the highspeed camera. The results of the tests are reported in Figure 19. We employed the dynamic parameters determined in Section VI.B, jointly with platform segment weights (reported in Table III) and flexible hinge rotational stiffness (reported in Section V.C) in the modelling of the pop-up platform. In the model, assuming all three legs have the same behavior, we considered a single leg elevating one third of the platform mass, in 
agreement with the considerations made in Section III.A. We report in Figure 20 the comparison of platform motion experimental and theoretical results.

From the experiment it was also evident that we could fine tune the platform speed by changing the voltage. The simulated results slightly overestimated platform performance by $15 \%$. This is likely due to the three legs that compose the system having some variability in dynamic parameters such as friction coefficients, and the coils' variability. These small differences made motion initiation slightly asynchronous thus reducing platform performance. To perfectly synchronize each leg, the powering parameters could be tuned according to each actuator's friction coefficient, inductance and resistance. One of the advantages of having a parallel platform with redundant actuation is the capability to maintain the top platform parallel to the base. We captured this feature in all the tests performed. The tests showed an average misalignment angle of $0.4 \pm 0.38^{\circ}$, thus demonstrating the accuracy of the fabrication method.

B. Load carrying capacity of the Pop-up platform

We evaluate the capability of the system by applying steady forces. We placed a force sensor (ATI Nano 17) at increasing distances from the top platform and after activation, we measured the steady forces. We repeated the tests at different voltages from 10 to $40 \mathrm{~V}$ and compared their results with simulations, as reported in Figure 21 . We obtained maximum steady forces of $0.17 \mathrm{~N}$; model predictions are more accurate for higher voltages, being less sensitive to friction forces. 
The last aspect that we considered was the workspace reduction due to the increase in payload. As an extension of the results reported in Section VII.A, we used the model to change platform payload and after fixing the minimum starting condition and voltage to generate motion, we calculated the final position and maximum achievable speed. As Figure 22 reports, an increase in the payload limits the platform's achievable speeds and its range of motion.

\section{CONCLUSIONS}

In this work we propose and prototype the design of a $1.7 \mathrm{~mm}$ thick pop-up portable platform that can repeatedly transform between a planar state to a 3D pop-up configuration. We advanced the SCM fabrication technique by embedding multiple essential components such as folding linkages and an actuation system in the different layers of the structure. We modeled and characterized the nominally 2-D electromagnetic actuation system, based on planar coils. Finally we identified the predominant physical effects influencing system behavior and compared experimental data and simulations for defining the design methodology and validating the models. We achieved maximum elevation of $13 \mathrm{~mm}$ and a maximum velocity of over $600 \mathrm{~mm} / \mathrm{s}$. The actuation time for performing the configuration change was lower than $0.1 \mathrm{~s}$. The proposed system lays the groundwork for new actuation in terms of performance, size and embedment of the actuation.

In the future we will implement semi-analytical models to improve the performance of magnetic forces calculation and to include the back electromagnetic 
force generated by the magnet moving on the coils. Other future challenges will be the embedment of position sensors and platform control. One of the advanced achievements expected will be the design of mechanisms with a higher number of DoFs. In particular, we chose the presented actuator configuration for an easy interface with delta robots and the envisioned system is shown in Figure 23.

Such a platform would generate interesting features, including inherent force control of the actuators and back-drivability of the transmission, thus making it ideal for HRI haptic applications or fragile object manipulation.

\section{ACKNOWLEDGMENTS}

The authors wish to thank Mr. Antoine Bartzen Spraurer from the École spéciale militaire de Saint-Cyr for his effort in planar coil manufacturing.

\section{FUNDING}

This work was supported by the Swiss NCCR Robotics in the framework of SpinFund project FOLDAWAY.

\section{REFERENCES}

[1] Wood RJ, Avadhanula S, Sahai R, Steltz E, Fearing RS (2008) Microrobot design using fiber reinforced composites. Journal of Mechanical Design. 130(5):052304.

[2] Felton S, Tolley M, Demaine E, Rus D, Wood R (2014) A method for building self-folding machines. Science 345(6197):644-6.

[3] Haldane DW, Casarez CS, Karras JT, Lee J, Li C, Pullin AO, Schaler EW, Yun D, Ota H, Javey A, Fearing RS (2015) Integrated manufacture of exoskeletons and sensing structures for folded millirobots. Journal of Mechanisms and Robotics, 7(2), p.021011 
[4] Koh JS, Cho KJ Omega-shaped inchworm-inspired crawling robot with large-index-andpitch (LIP) SMA spring actuators (2013) Mechatronics, IEEE/ASME Transactions on 18(2):419-29.

[5] Firouzeh A, Paik J (2015) A Fully Integrated Low-Profile Robotic Origami (2015) ASME. J. Mechanisms Robotics 7(2):021009-021009-8. doi:10.1115/1.4029491.

[6] Noh M, Kim SW, An S, Koh JS, Cho KJ (2012) Flea-inspired catapult mechanism for miniature jumping robots. Robotics, IEEE Transactions on 28(5):1007-18.

[7] Zhakypov Z, Falahi M, Shah M, Paik J (2015) The design and control of the multi-modal locomotion origami robot, Tribot. In Intelligent Robots and Systems (IROS), 2015 IEEE/RSJ International Conference on (pp. 4349-4355).

[8] Wood RJ (2008) The first takeoff of a biologically inspired at-scale robotic insect. Robotics, IEEE Transactions on 24(2):341-7.

[9] Salerno, Marco, Ketao Zhang, Arianna Menciassi, and Jian S. Dai. "A novel 4-DOF origami grasper with an SMA-actuation system for minimally invasive surgery." IEEE Transactions on Robotics 32, no. 3 (2016): 484-498.

[10] Zhang, Ketao, Chen Qiu, and Jian S. Dai. "An extensible continuum robot with integrated origami parallel modules." Journal of Mechanisms and Robotics 8, no. 3 (2016): 031010.

[11] Zhang, Ketao, Jian S. Dai, and Yuefa Fang. "Topology and constraint analysis of phase change in the metamorphic chain and its evolved mechanism." Journal of Mechanical Design 132, no. 12 (2010): 121001.

[12] Zhang, Ketao, and Jian S. Dai. "A kirigami-inspired 8r linkage and its evolved overconstrained $6 r$ linkages with the rotational symmetry of order two." Journal of Mechanisms and Robotics 6, no. 2 (2014): 021007.

[13] Baisch AT, Sreetharan PS, Wood RJ (2010) Biologically-inspired locomotion of a 2g hexapod robot. In Intelligent Robots and Systems (IROS), 2010 IEEE/RSJ International Conference on (pp. 5360-5365).

[14] Diller E, Pawashe C, Floyd S, Sitti M. (2011) Assembly and disassembly of magnetic mobile micro-robots towards deterministic 2-D reconfigurable micro-systems. The International Journal of Robotics Research, 30 (14): 1667-1680.

[15] Reissman T, Park JS, Garcia E. (2012) Multilayer, Stacked Spiral Copper Inductors on Silicon with Micro-Henry Inductance Using Single-Level Lithography. Active and Passive Electronic Components. 
[16] Sun X, Zheng Y, Li Z, Li X, Zhang H (2013) Stacked flexible parylene-based 3D inductors with $\mathrm{Ni} 80 \mathrm{Fe} 20$ core for wireless power transmission system. In Micro Electro Mechanical Systems (MEMS), 2013 IEEE 26th International Conference on (pp. 849-852).

[17] Meyer CD, Bedair SS, Morgan BC, Arnold DP (2012) Influence of layer thickness on the performance of stacked thick-film copper air-core power inductors. Magnetics, IEEE Transactions on 48(11):4436-9.

[18] Futai N, Matsumoto K, Shimoyama I (2004) A flexible micromachined planar spiral inductor for use as an artificial tactile mechanoreceptor. Sensors and Actuators A: Physical 111(2):293-303.

[19] Takahashi H, DOHI T, Matsumoto K, Shimoyama I (2007) A micro planar coil for local high resolution magnetic resonance imaging. In Micro Electro Mechanical Systems, 2007. MEMS. IEEE 20th International Conference on (pp. 549-552).

[20] Kim KH, Yoon HJ, Jeong OC, Yang SS (2005) Fabrication and test of a micro electromagnetic actuator. Sensors and Actuators A: Physical 117(1):8-16.

[21] Zárate JJ, Tosolini G, Petroni S, De Vittorio M, Shea H (2015) Optimization of the force and power consumption of a microfabricated magnetic actuator. Sensors and Actuators A: Physical 234:57-64.

[22] Cugat O, Basrour S, Divoux C, Mounaix P, Reyne G (2001) Deformable magnetic mirror for adaptive optics: technological aspects. Sensors and Actuators A: Physical 89(1):1-9.

[23] Benali-Khoudja M, Hafez M, Kheddar A (2007) VITAL: an electromagnetic integrated tactile display. Displays 28(3):133-44.

[24] Chang PJ, Chang FW, Yuen MC, Otillar R, Horsley DA (2014) Force measurements of a magnetic micro actuator proposed for a microvalve array. Journal of Micromechanics and Microengineering 24(3):034005.

[25] Gieras JF, Piech ZJ, Tomczuk B (2011) Linear synchronous motors: transportation and automation systems. CRC press.

[26] Cugat O, Delamare J, Reyne G. (2003) Magnetic micro-actuators and systems (MAGMAS). Magnetics, IEEE Transactions on. 39(6):3607-12.

[27] Niarchos D. (2003) Magnetic MEMS: key issues and some applications. Sensors and Actuators A: Physical 109(1):166-73.

[28] Comsol (2011) AB. AC/DC Module-User's Guide. COMSOL.;3:151. 
[29] Firouzeh A, Sun Y, Lee H, Paik J (2013) Sensor and actuator integrated low-profile robotic origami. In Intelligent Robots and Systems (IROS), 2013 IEEE/RSJ International Conference on (pp. 4937-4944).

[30] Grover FW (2004) Inductance calculations: working formulas and tables. Courier Corporation.

[31] Kasap SO (2006) Principles of electronic materials and devices. McGraw-Hill.

[32] Qiu, Chen, Ketao Zhang, and Jian S. Dai. "Repelling-Screw Based Force Analysis of Origami Mechanisms." Journal of Mechanisms and Robotics 8, no. 3 (2016): 031001. 


\section{Figures Caption list}

Fig. 1. Parallel mechanism designed as a proof-of-concept of the foldable actuation mechanism. (Left), the unfolded 2D configuration top and side view, (Right) 3Dfolded configuration upon actuation, top and side view. The arrows mark the direction of motion of the platform components after coil powering.

Fig. 2. Miniaturized electromagnetic actuator. Working principle and main components, the red arrows mark the magnetization direction of magnet and coils.

Fig. 3. Schematic of the layers composing the platform (left). Assembled system in the unfolded 2D state (center), folded 3D system configuration (right).

Fig. 4. Double slider mechanism: The proposed platform is axially symmetric and composed of 3 double sliders arranged at $120^{\circ}$ from one another and sharing the prismatic joint.

Fig. 5. Working principle of the miniaturized electromagnetic actuator. (a) Schematic of the assembled actuator and (b) Exploded view of the layers composing the actuator

Fig. 6. Magnet-coils interactions. The reference system used to define the position of the magnet with respect to the coils (a). Resultant force acting on the magnet with increasing $\mathrm{X}$ positions (b). Resulting FEM forces in $\mathrm{X}$ and $\mathrm{Z}$ directions by changing magnet $\mathrm{X}$ position (c-d). Resulting FEM forces in X direction by changing magnet $\mathrm{X}$ position and magnet elevation along $\mathrm{Z}$ (e). Resulting FEM forces in $\mathrm{Z}$ direction by changing magnet $X$ position and magnet elevation along $Z(f)$.

Fig. 7. Schematics of the articulated parallel platform in pop-up (left), and planar 
(right), configurations with design parameters used. The values of the parameters used are reported in Table II.

Fig. 8. Mask and coil prototypes. Comparison of masks (a-b), corresponding resulting coils (c-d). In the manufactured coil, the under-etching effect is visible; conductor tracks are much thinner than the corresponding mask tracks.

Fig. 9. Platform prototype: The alignment holes used during the fabrication process are visible in both pictures. The assembled system is in the 2D state (left), and in the pop-up state (right).

Fig. 10. Platform prototype sizes. The weights of the components and sub components highlighted in the figure are reported in Table III.

Fig. 11. Free body diagrams of the three links composing the double slider mechanism

Fig. 12. Description of experimental setups and tests performed. The setup for the verification of the coils' time constant is composed of a Hall-effect sensor and the coil system (a). The setup for verification of the single rail dynamics is composed of a high-speed camera and a tracking marker (b).

Fig. 13. Magnetic field development with time: for actuation voltages of 20 and $30 \mathrm{~V}$, verifying negligible electromagnetic transient response.

Fig. 14. Normalized magnetic flux density decrease due to thermal increase. Simulation (a), Experimental (b). B field expected decrease per second for different input voltages (c). 
Fig. 15. The slider's position on the rail: The Fx for different positions along the rail is reported (a). For different voltages, the magnet position in time is reported (b). Calculated parameters used in the comparison with the model (c).

Fig. 16. Magnet motion calculated parameters comparison: maximum speed (a), maximum position (b), final position (c), and motion time (d). Simulations (in red) and experiments (in blue), the error bars are calculated from the standard deviation of three repetitions.

Fig. 17. Description of experimental setups and tests performed: The setup for the verification of the platform dynamics includes a marker on each leg (a). The setup for the payload tests includes a mass placed on top of the platform (b).

Fig. 18. The pop-up platform's tracking performance. At the different voltages we report the top platform position (a), speed (b) and acceleration (c).

Fig. 19. Pop-up platform with a mass on top: For different weights, we report the top platform position (a), speed (b) and acceleration (c). We report the minimum elevation and powering voltage to initiate motion in the legend.

Fig. 20. Pop-up platform calculated motion parameters comparison: maximum speed (a), maximum position (b), final position (c) and motion time (d). Comparison of the experimental (blue), and simulated (red), results. The trends are very similar and the simulation captures well platform motion.

Fig. 21. Legged-system steady forces tests. Experimental setup (a) includes a force sensor and a micro stage placed on top of the platform. The comparison between experimental (blue) and theoretical (red) results is reported (b). 
Fig. 22. Pop-up platform with mass on top, simulation results. For different weights and input voltages, the top platform achievable position ranges (a) and speeds (b) are reported.

Fig. 23. Envisioned platform where a delta robot is interfaced with the proposed actuation system. Folded portable configuration (left), 3D pop-up configuration (right). 
Figures

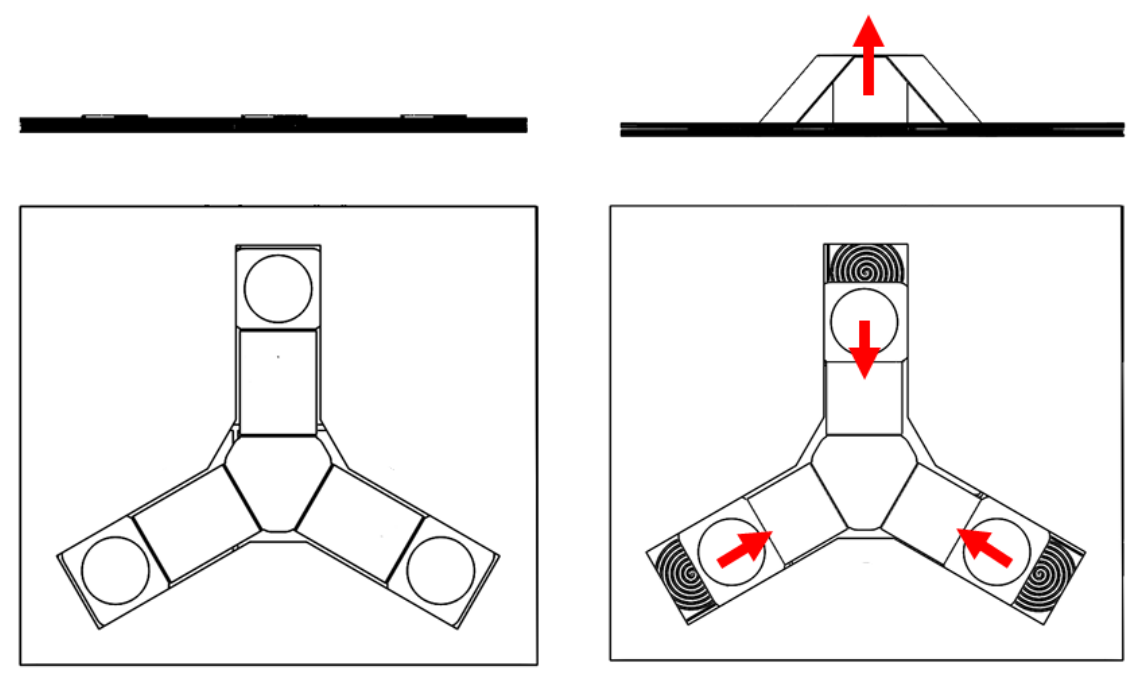

Fig. 1. Parallel mechanism designed as a proof-of-concept of the foldable actuation mechanism. (Left), the unfolded 2D configuration top and side view, (Right) 3D-folded configuration upon actuation, top and side view. The arrows mark the direction of motion of the platform components after coil powering. 


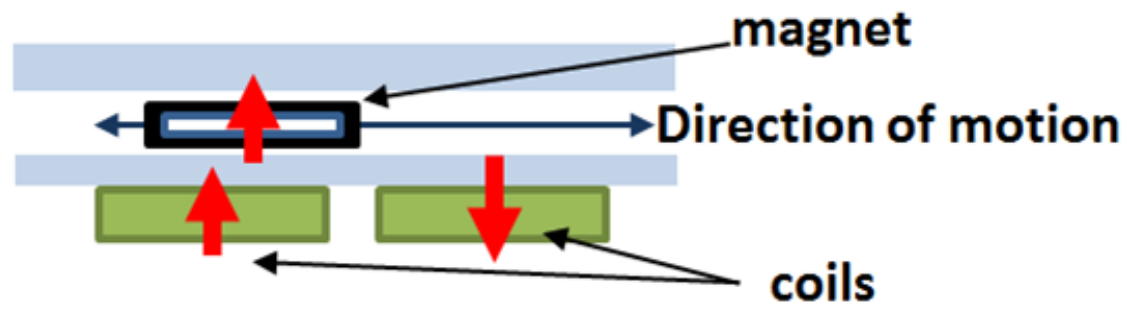

Fig. 2. Miniaturized electromagnetic actuator. Working principle and main components, the red arrows mark the magnetization direction of magnet and coils. 

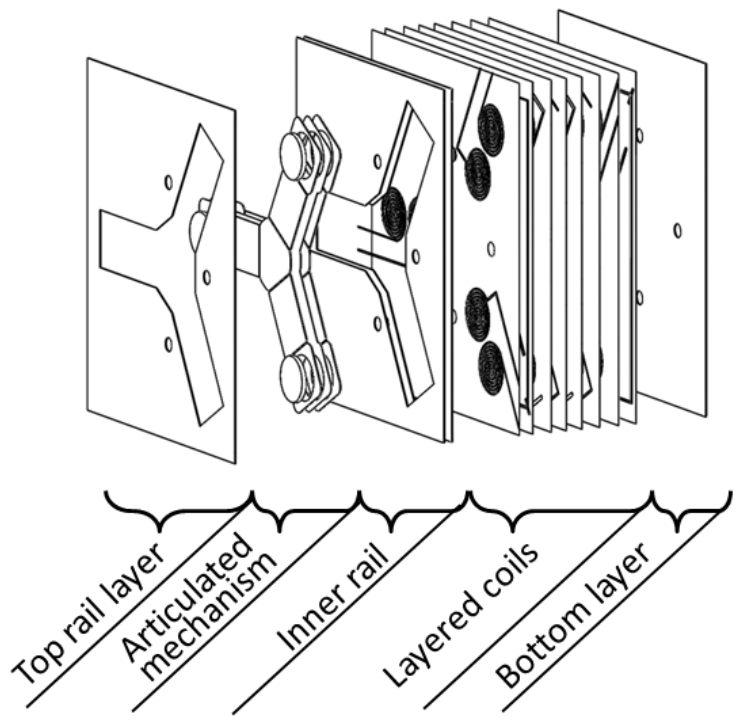

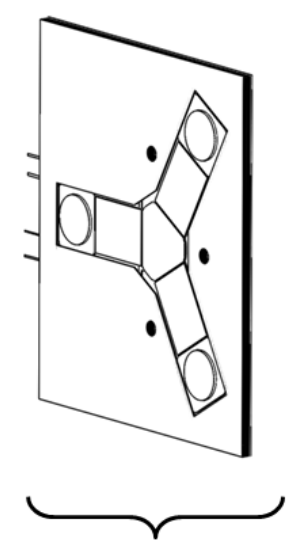

Assembled system in planar configuration

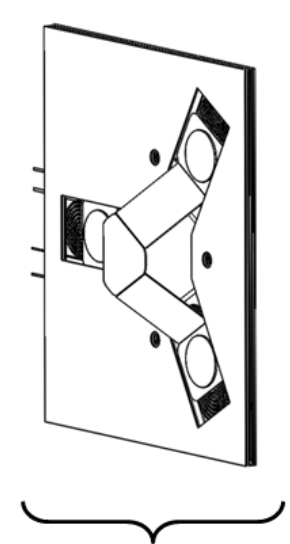

Assembled system in 3D configuration

Fig. 3. Schematic of the layers composing the platform (left). Assembled system in the unfolded 2D state (center), folded 3D system configuration (right). 


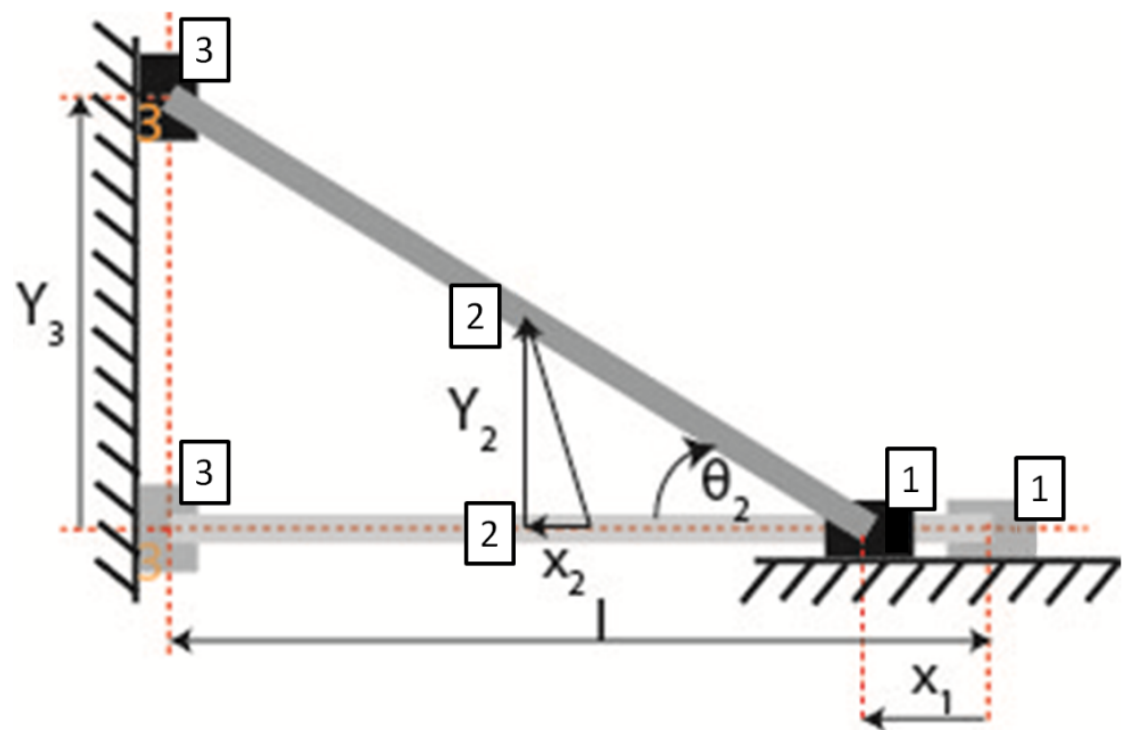

Fig. 4. Double slider mechanism: The proposed platform is axially symmetric and composed of 3 double sliders arranged at $120^{\circ}$ from one another and sharing the prismatic joint. 


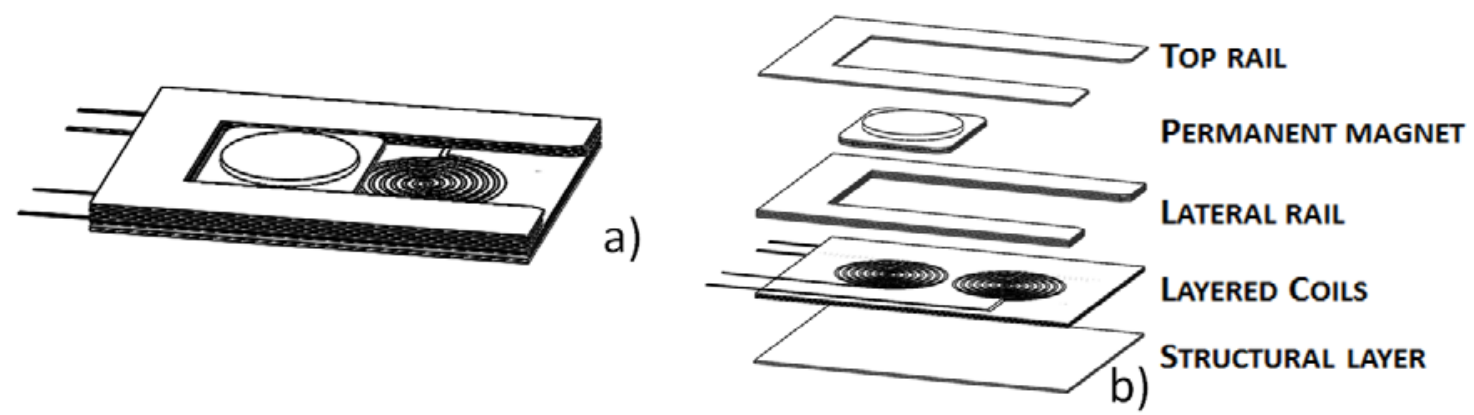

Fig. 5. Working principle of the miniaturized electromagnetic actuator. (a) Schematic of the assembled actuator and (b) Exploded view of the layers composing the actuator 


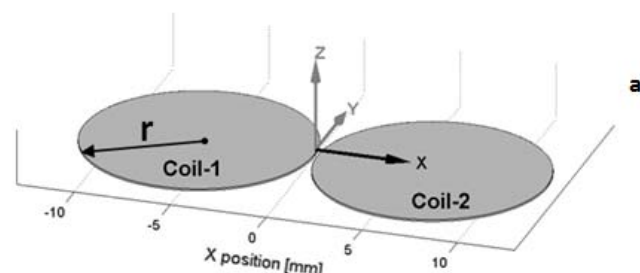

a)
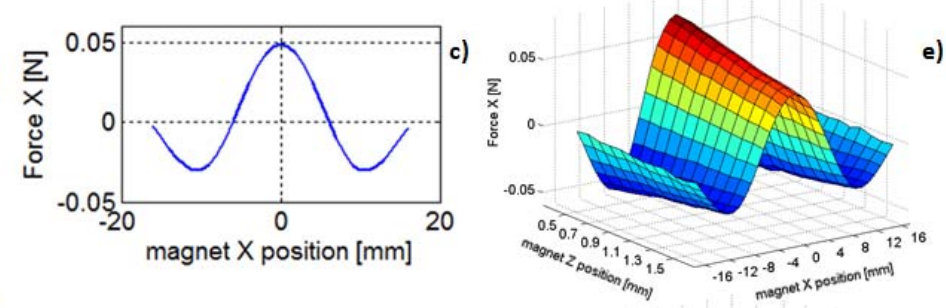

e)

b)
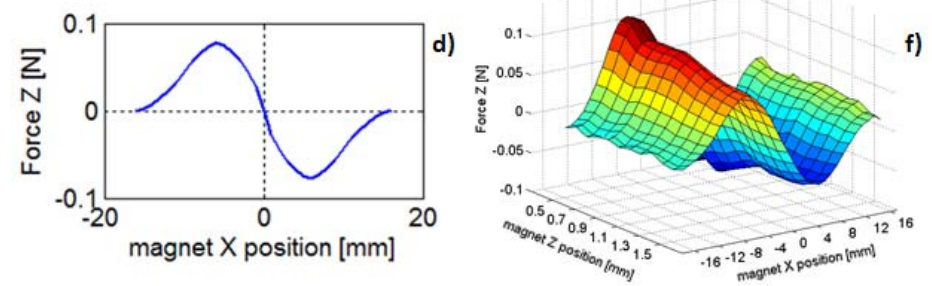

Fig. 6. Magnet-coils interactions. The reference system used to define the position of the magnet with respect to the coils (a). Resultant force acting on the magnet with increasing X positions (b). Resulting FEM forces in $\mathrm{X}$ and $\mathrm{Z}$ directions by changing magnet $\mathrm{X}$ position (c-d). Resulting FEM forces in $X$ direction by changing magnet $X$ position and magnet elevation along $Z(e)$. Resulting FEM forces in $Z$ direction by changing magnet $X$ position and magnet elevation along $Z$ ( $f$ ). 


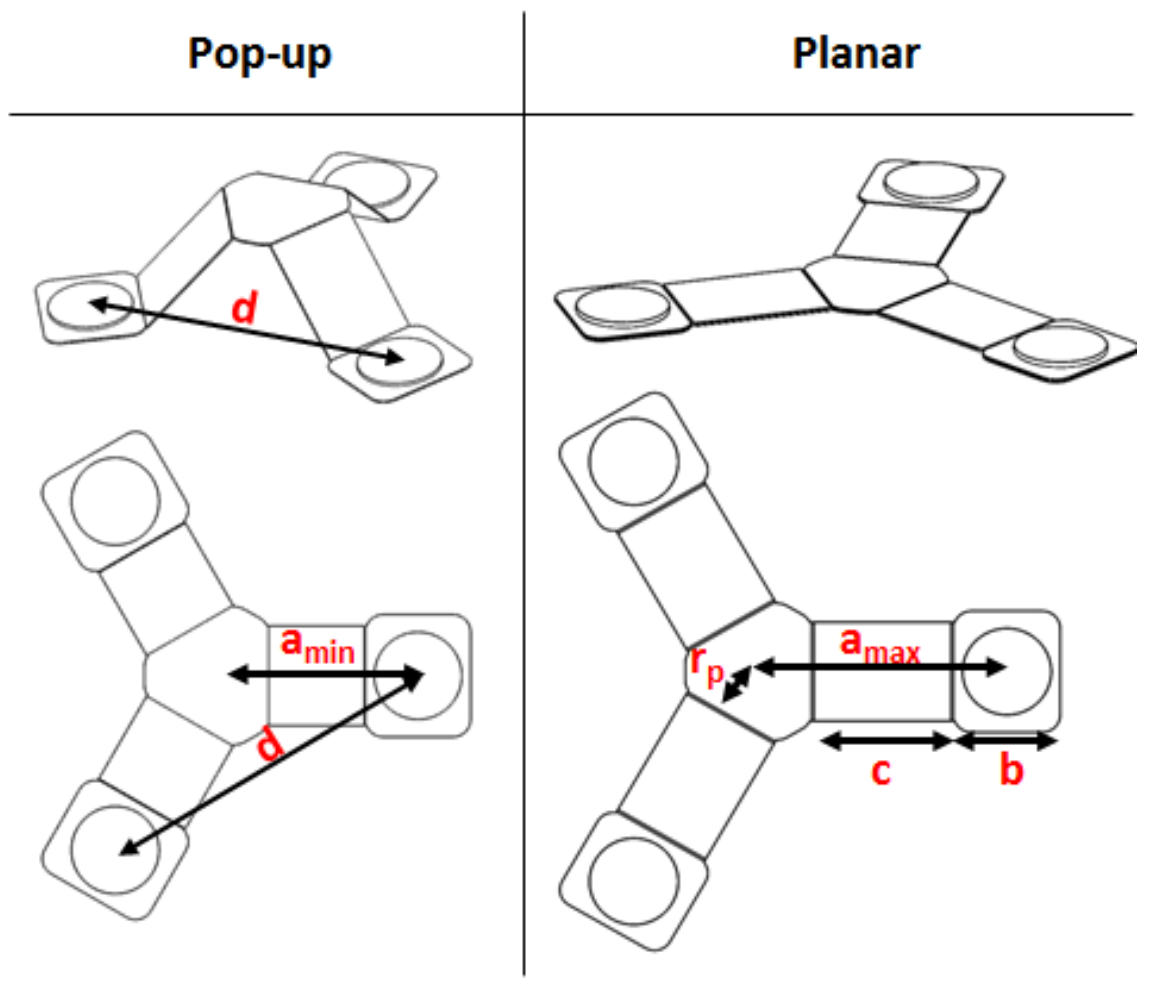

Fig. 7. Schematics of the articulated parallel platform in pop-up (left), and planar (right), configurations with design parameters used. The values of the parameters used are reported in Table II. 


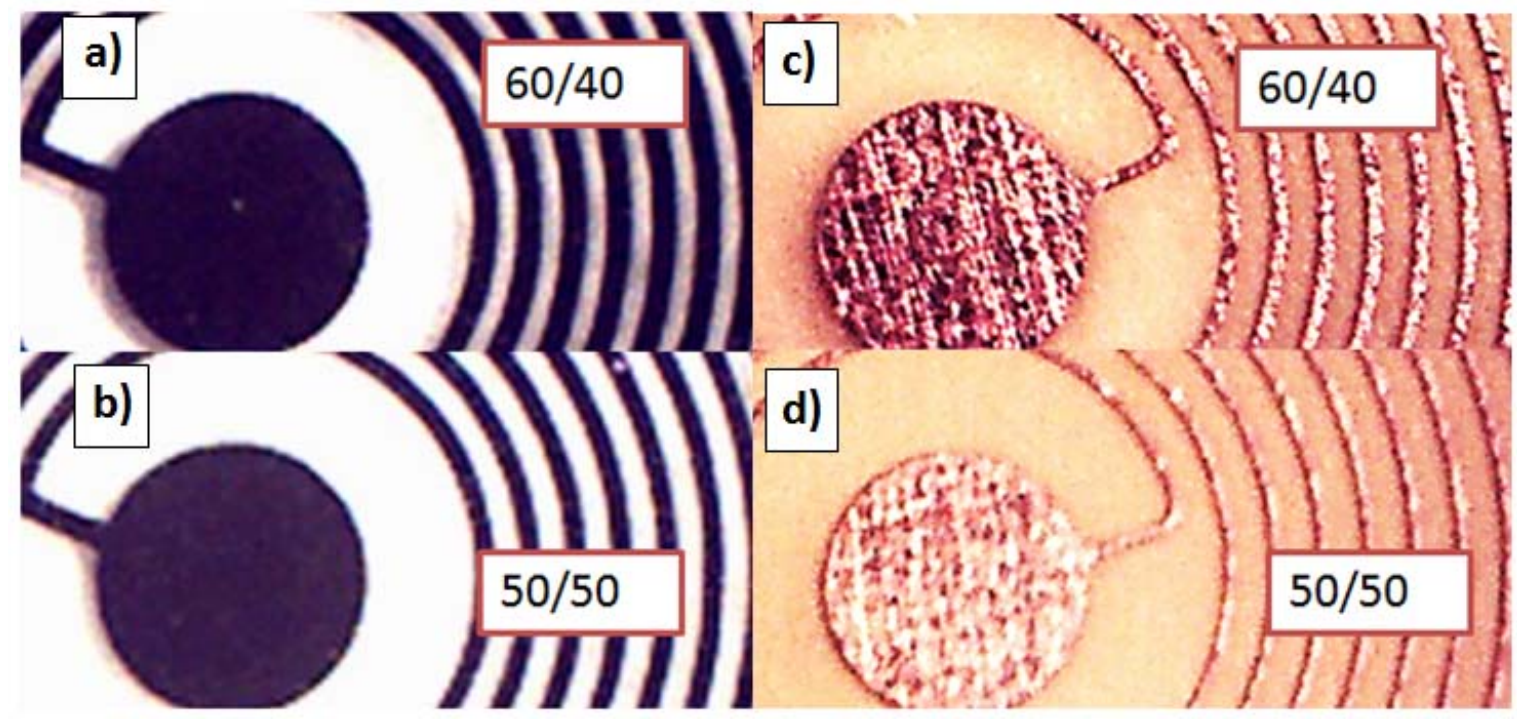

Fig. 8. Mask and coil prototypes. Comparison of masks (a-b), corresponding resulting coils (c-d). In the manufactured coil, the under-etching effect is visible; conductor tracks are much thinner than the corresponding mask tracks. 

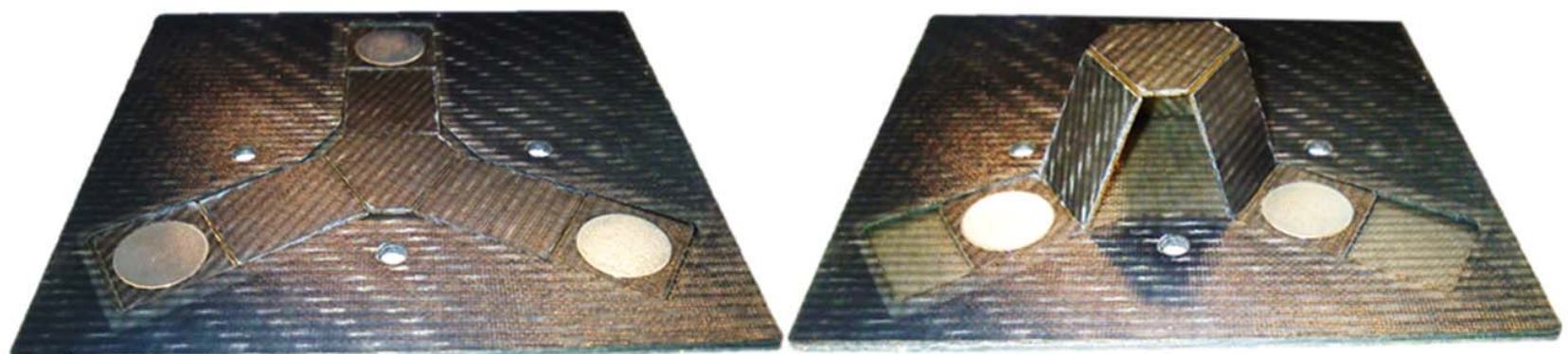

$20 \mathrm{~mm}$

Fig. 9. Platform prototype: The alignment holes used during the fabrication process are visible in both pictures. The assembled system is in the $2 \mathrm{D}$ state (left), and in the pop-up state (right). 


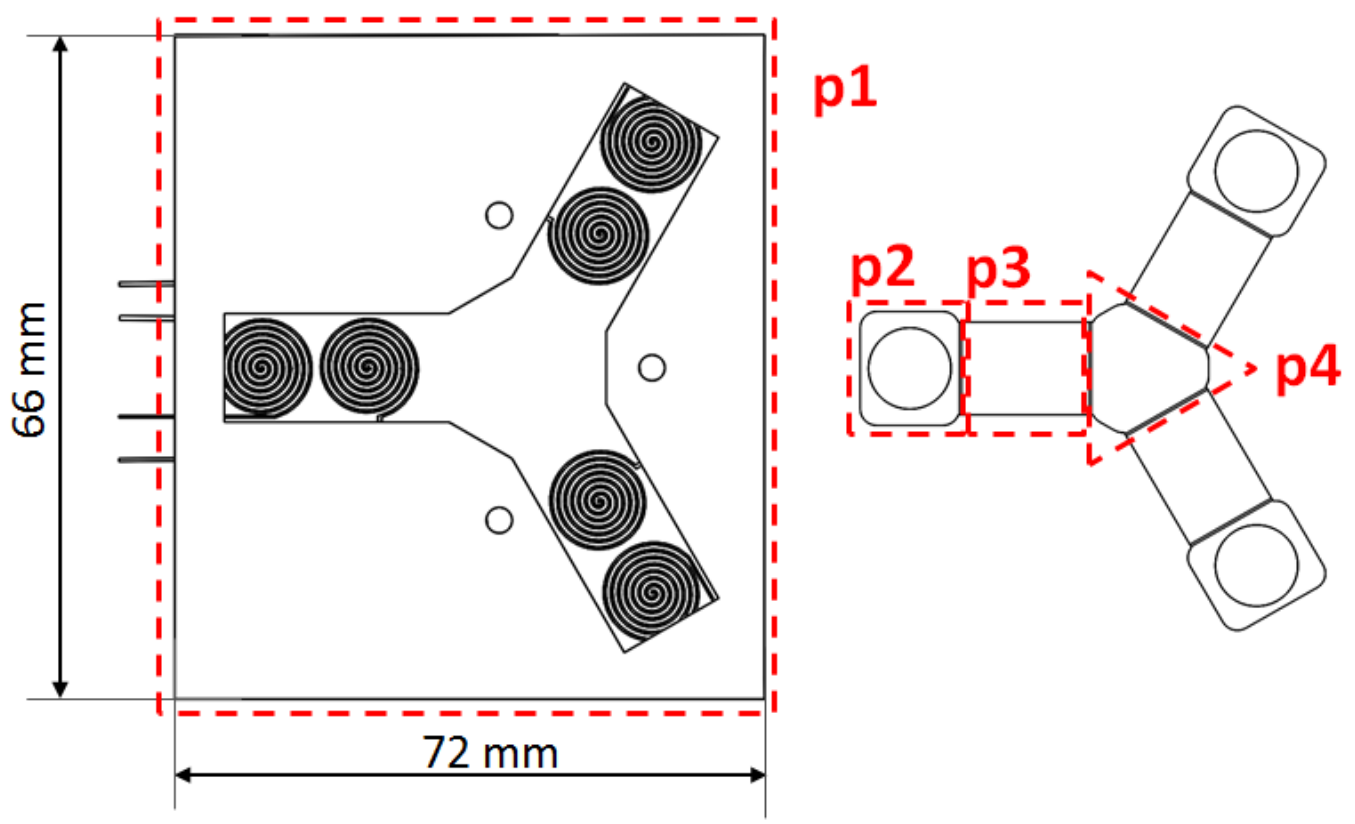

Fig. 10. Platform prototype sizes. The weights of the components and sub components highlighted in the figure are reported in Table III. 
ASME Journal of Mechanisms and Robotics
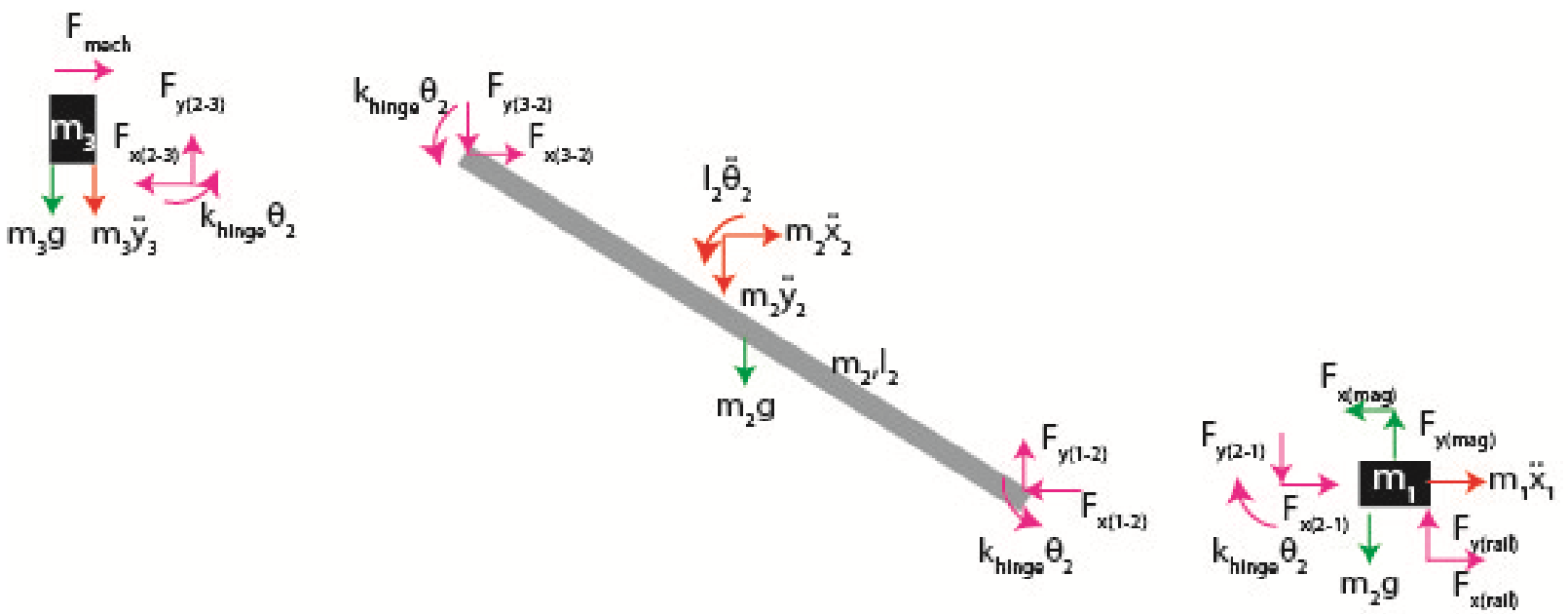

Fig. 11. Free body diagrams of the three links composing the double slider mechanism 
a)

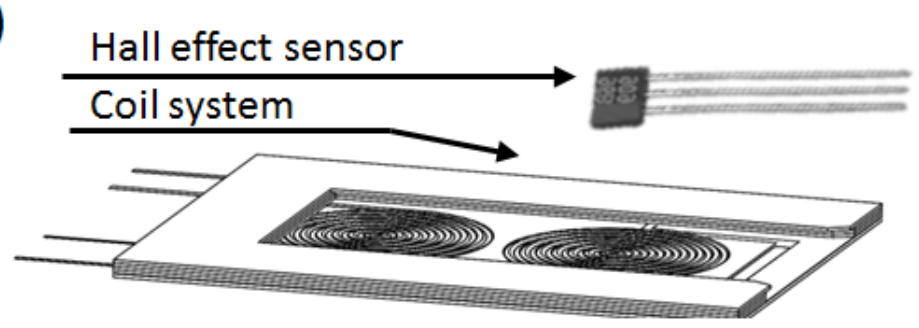

b)

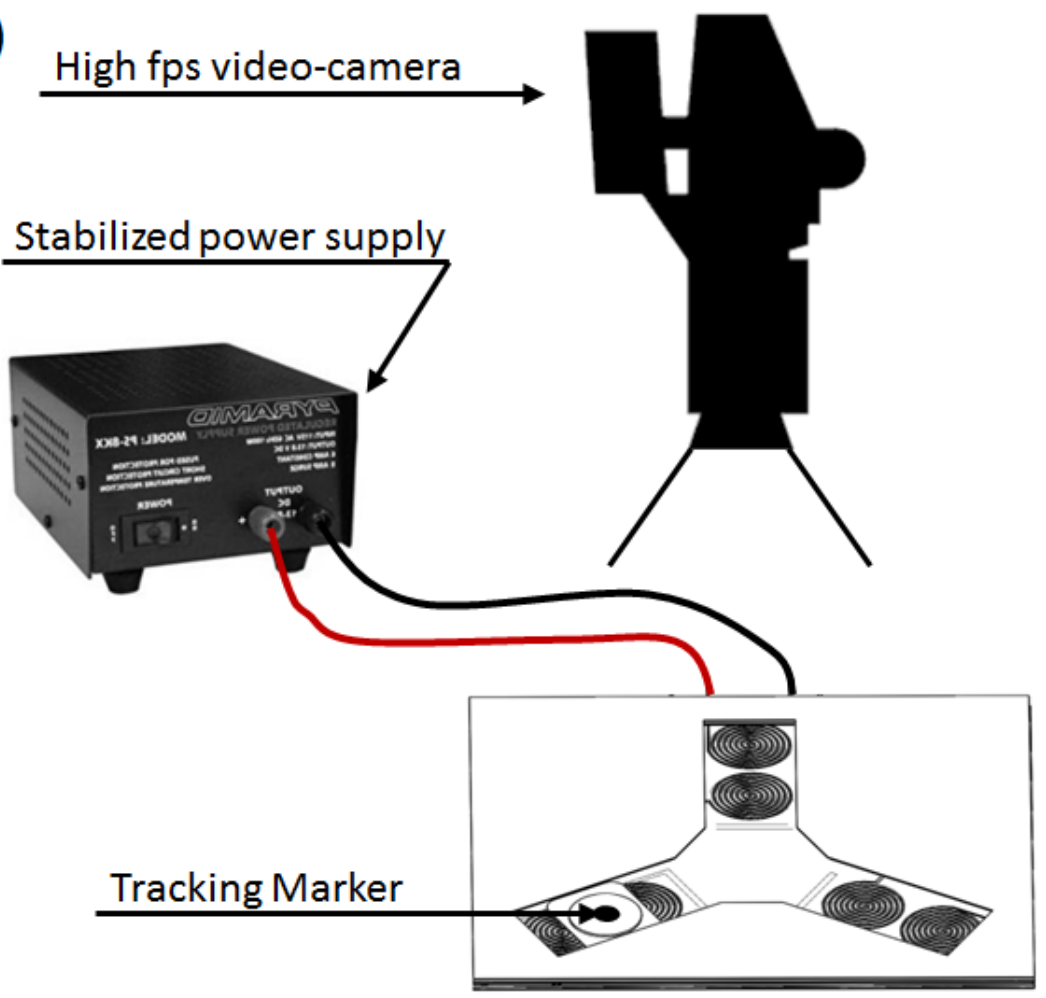

Fig. 12. Description of experimental setups and tests performed. The setup for the verification of the coils' time constant is composed of a Hall-effect sensor and the coil system (a). The setup for verification of the single rail dynamics is composed of a highspeed camera and a tracking marker (b). 


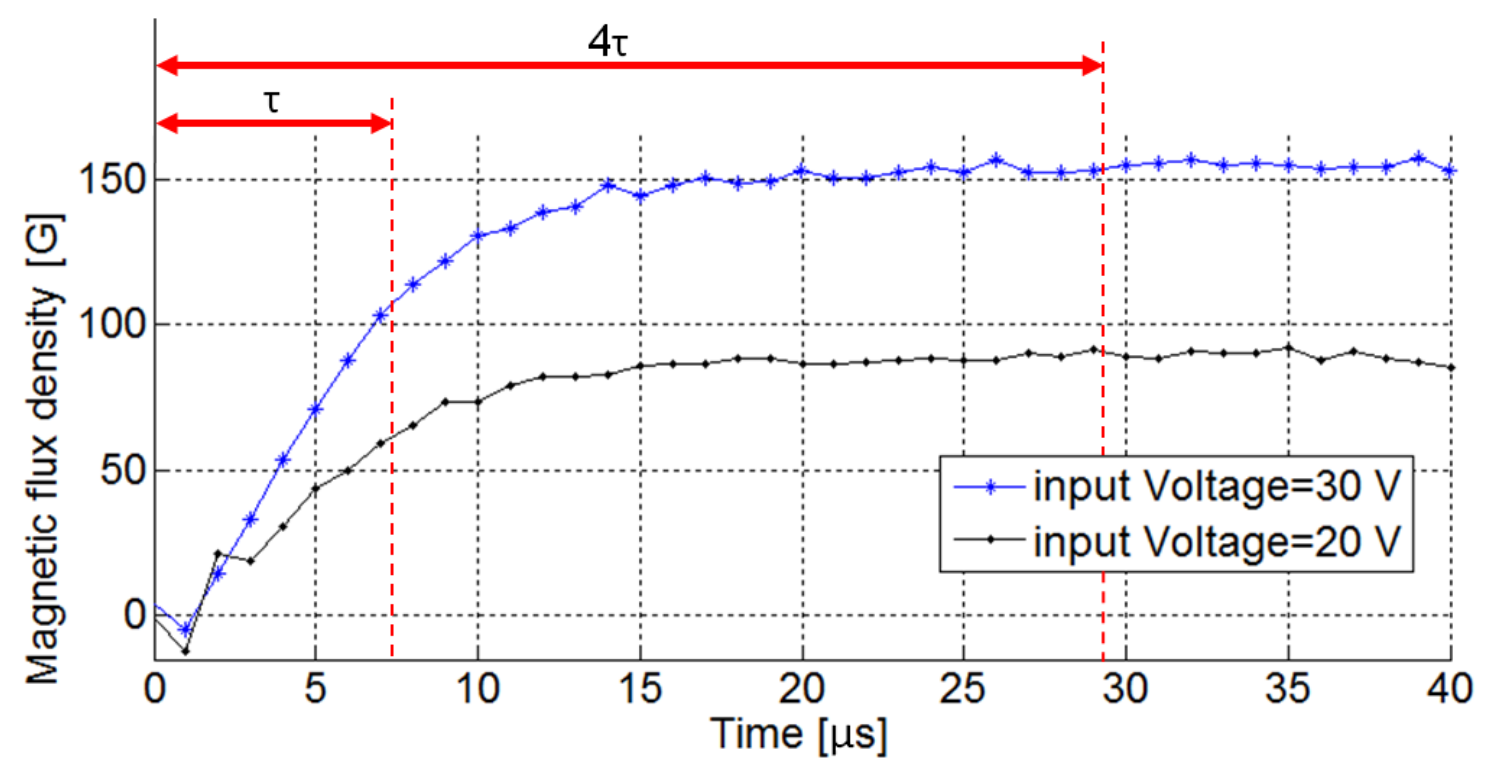

Fig. 13. Magnetic field development with time: for actuation voltages of 20 and $30 \mathrm{~V}$, verifying negligible electromagnetic transient response. 
ASME Journal of Mechanisms and Robotics
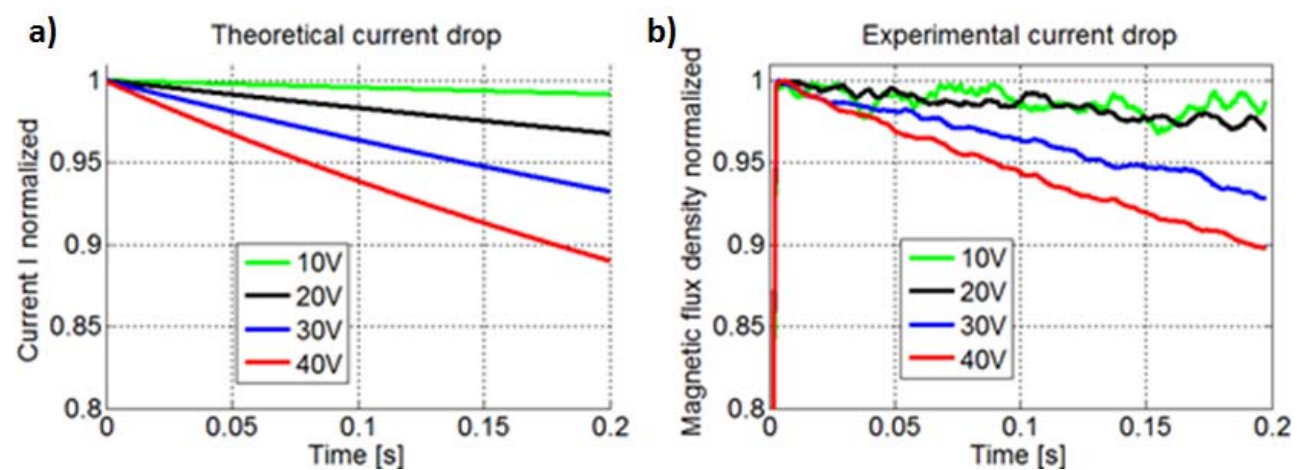

c) $\bar{\omega}$

B field decrease rate for different Voltages

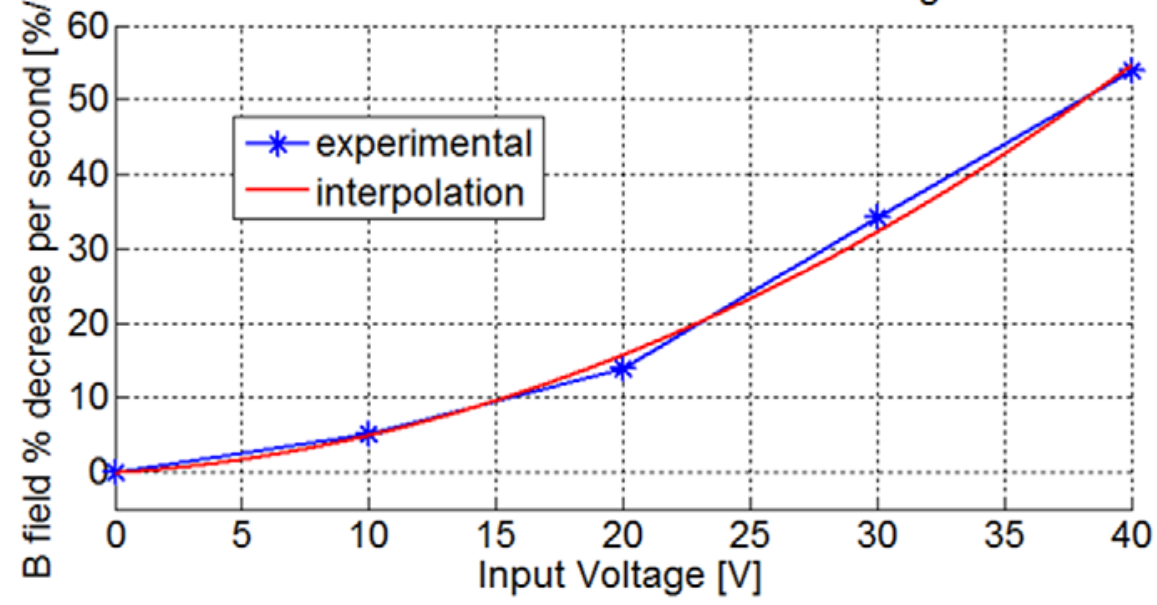

Fig. 14. Normalized magnetic flux density decrease due to thermal increase. Simulation

(a), Experimental (b). B field expected decrease per second for different input voltages

(c). 

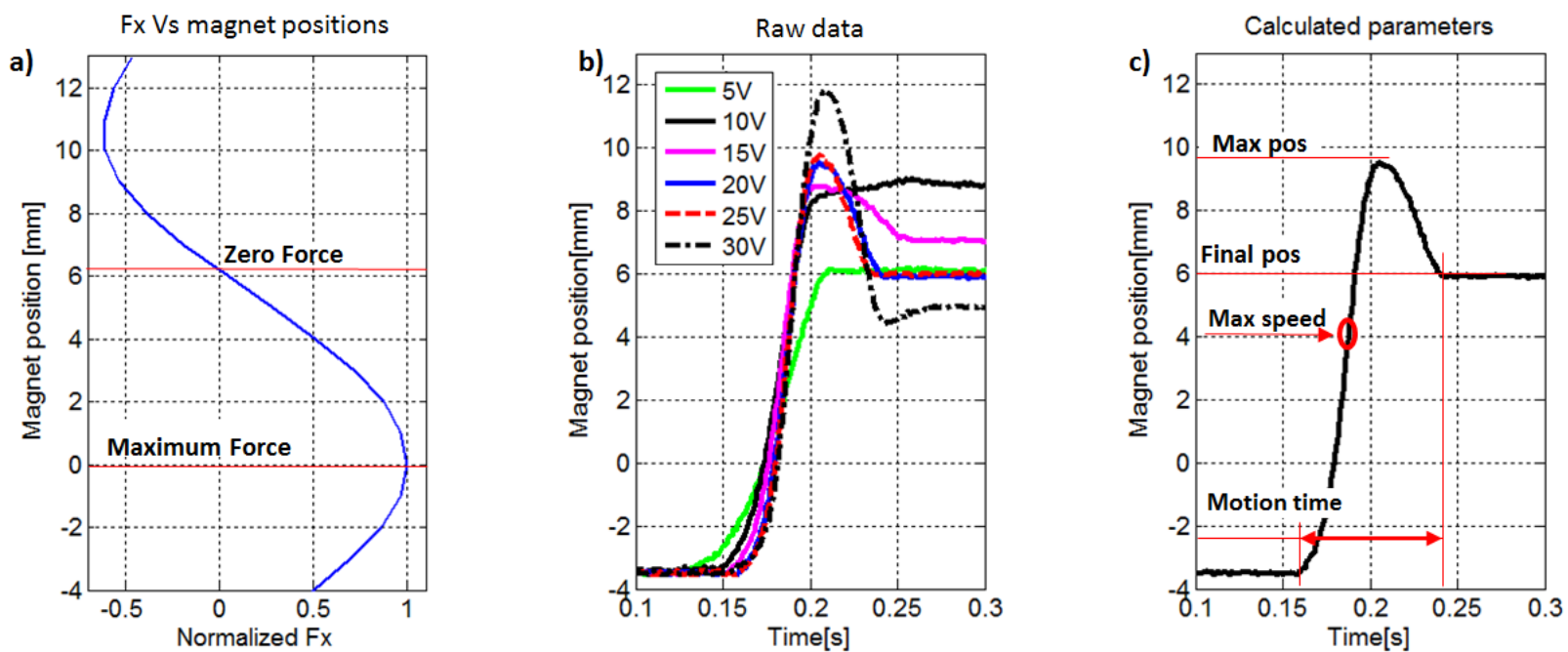

Fig. 15. The slider's position on the rail: The Fx for different positions along the rail is reported (a).

For different voltages, the magnet position in time is reported (b). Calculated parameters used in the comparison with the model (c). 

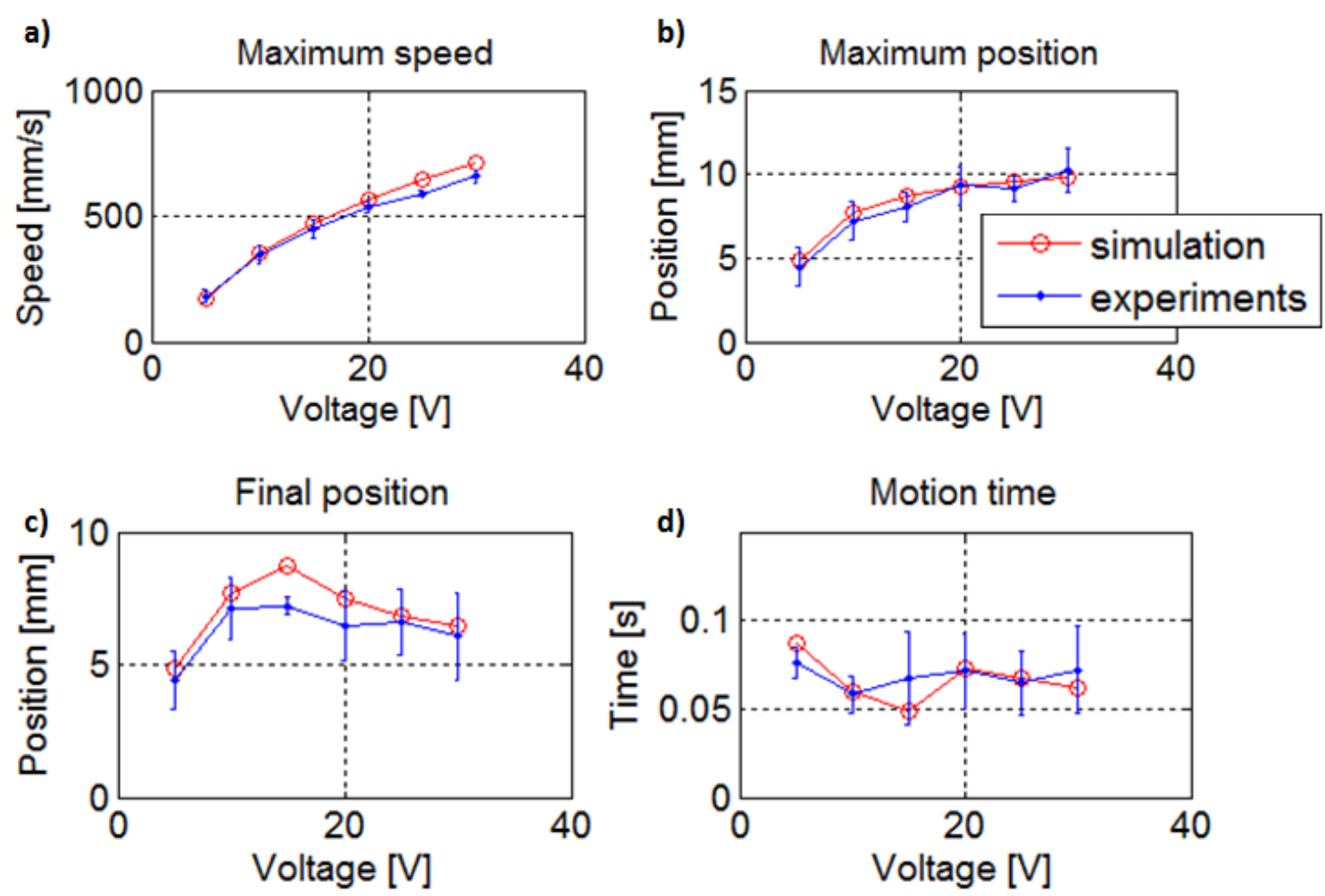

Fig. 16. Magnet motion calculated parameters comparison: maximum speed (a), maximum position (b), final position (c), and motion time (d). Simulations (in red) and experiments (in blue), the error bars are calculated from the standard deviation of three repetitions. 


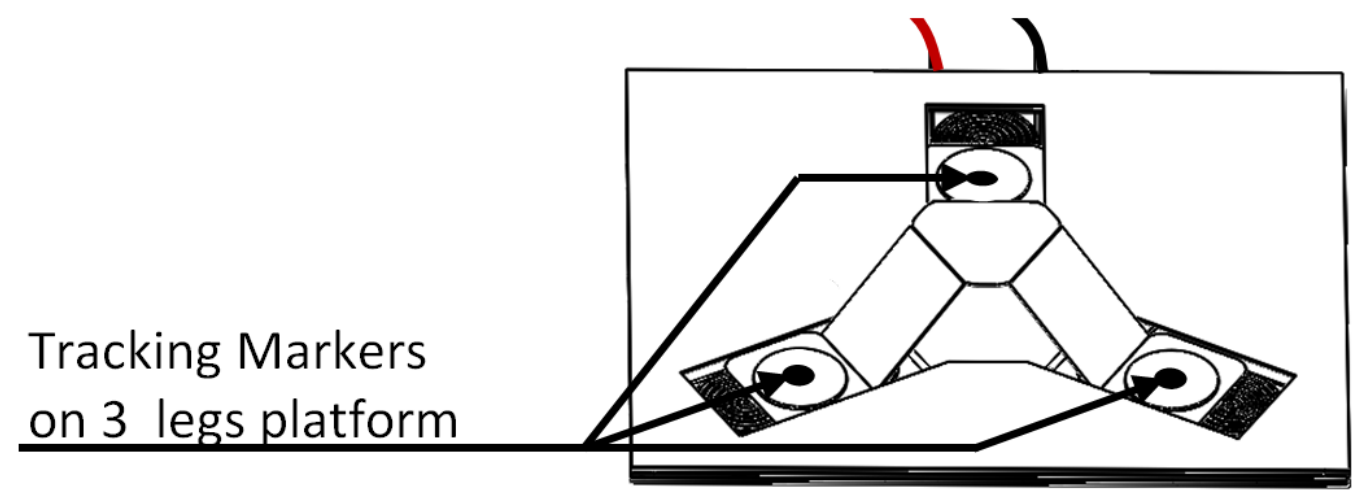

a)

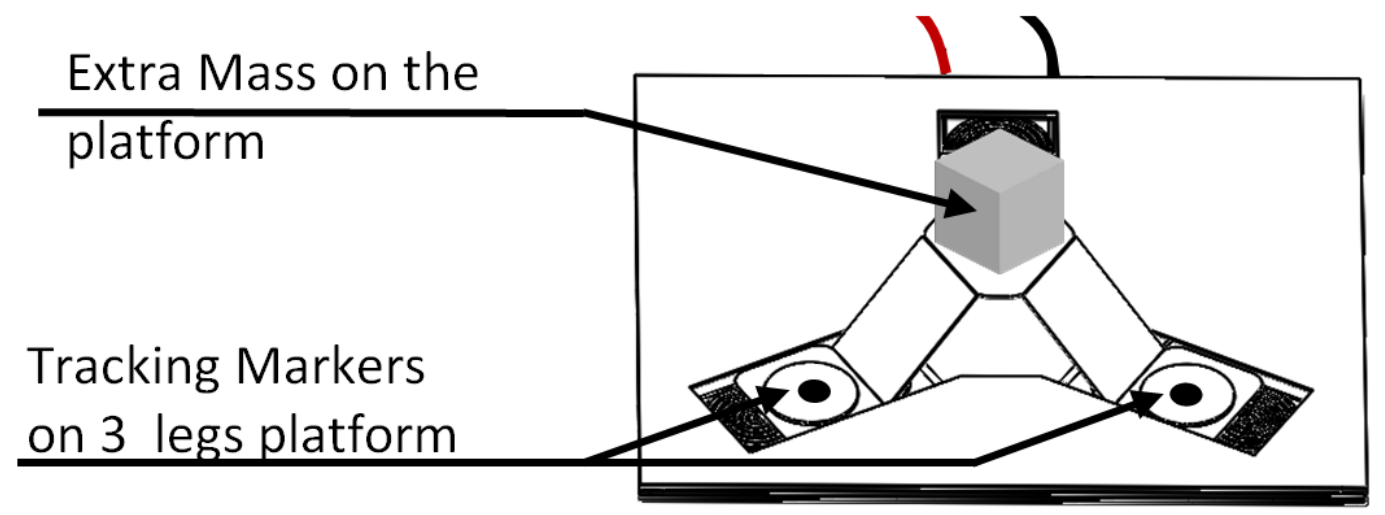

b)

Fig. 17. Description of experimental setups and tests performed: The setup for the verification of the platform dynamics includes a marker on each leg (a). The setup for the payload tests includes a mass placed on top of the platform (b). 

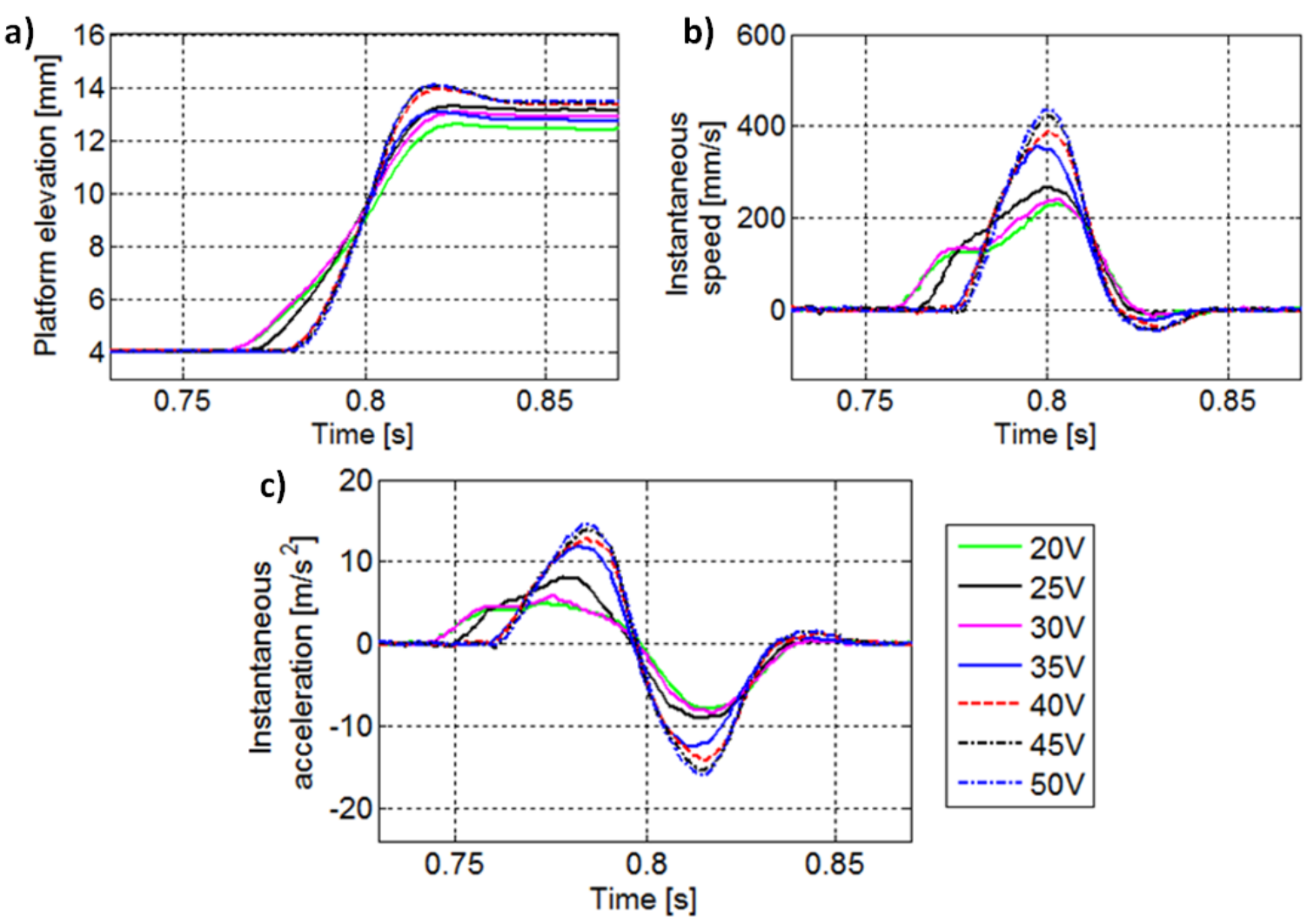

Fig. 18. The pop-up platform's tracking performance. At the different voltages we report the top platform position (a), speed (b) and acceleration (c). 
a)

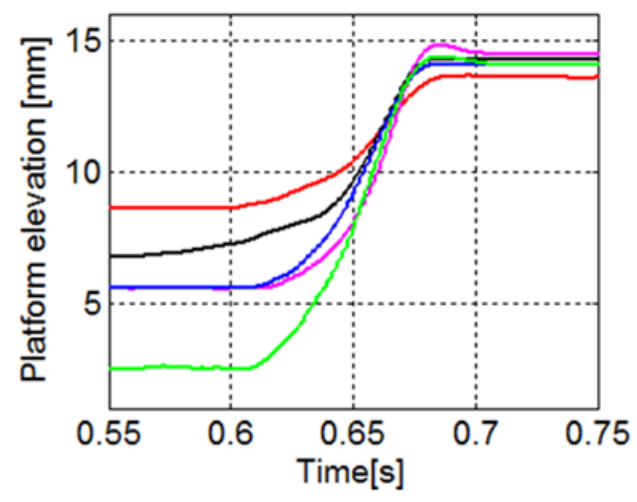

c)

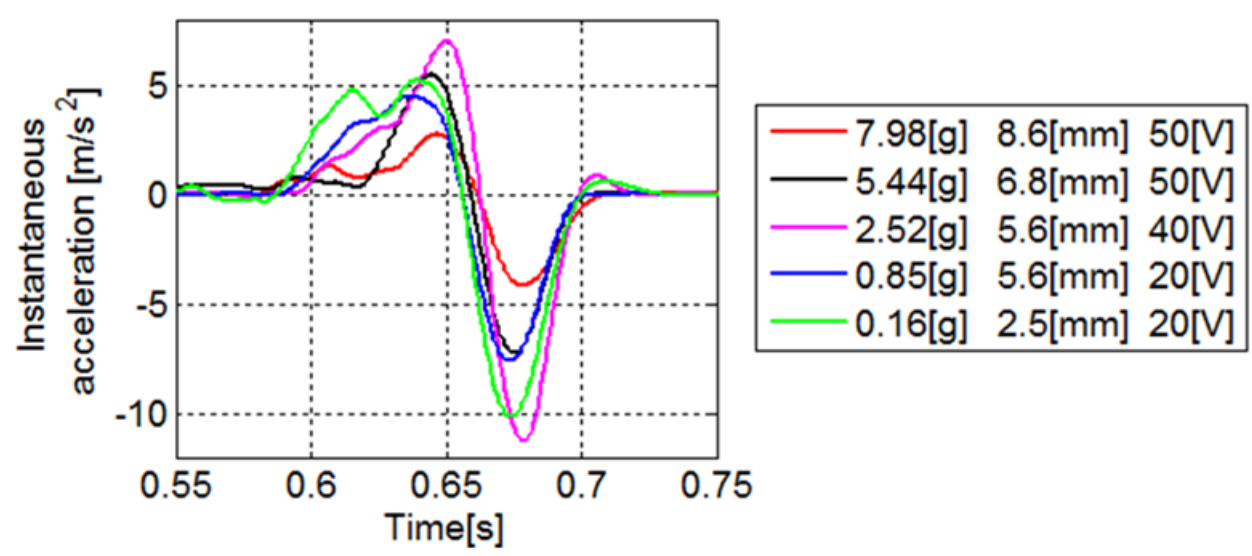

Fig. 19. Pop-up platform with a mass on top: For different weights, we report the top platform position (a), speed (b) and acceleration (c). We report the minimum elevation and powering voltage to initiate motion in the legend. 

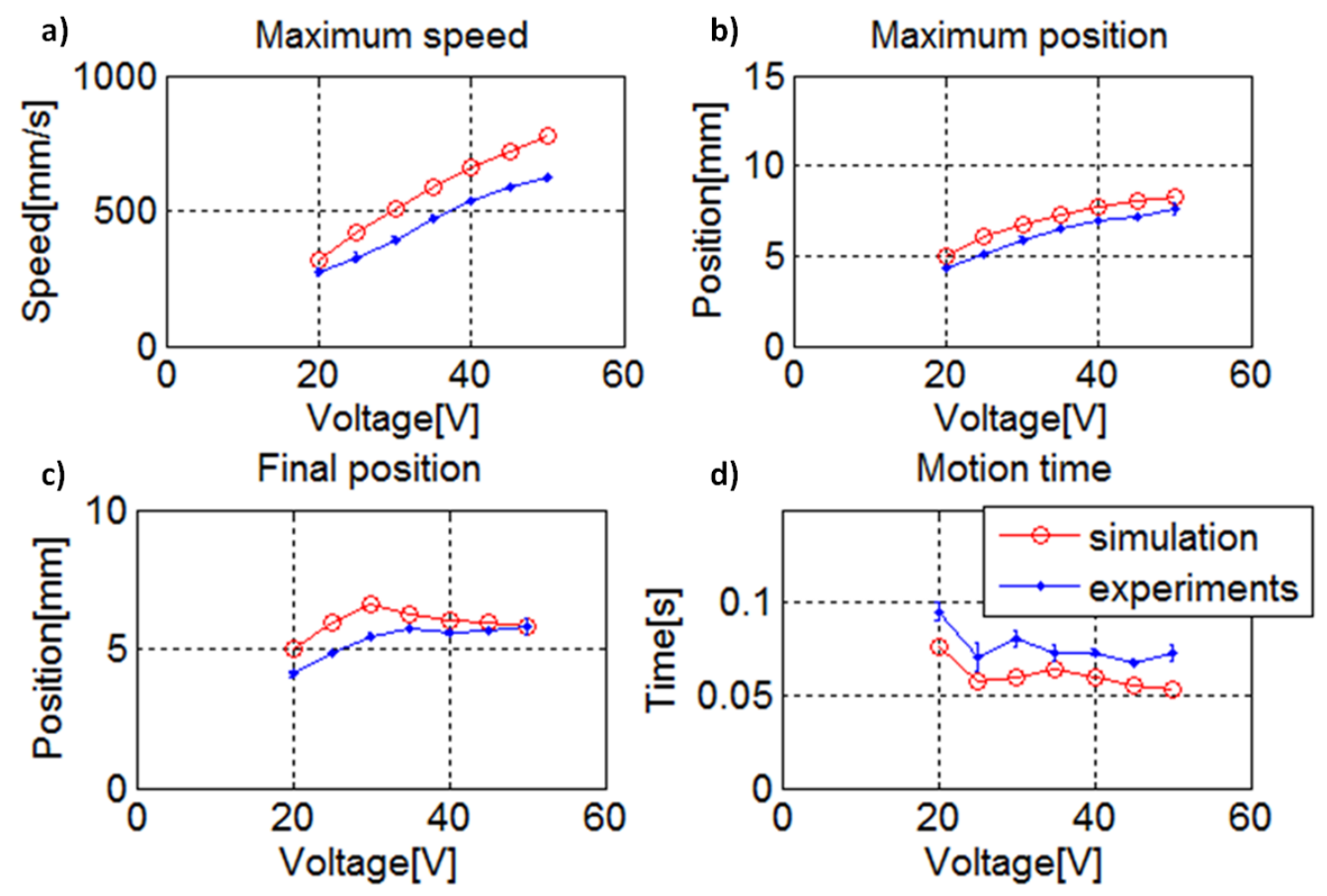

Fig. 20. Pop-up platform calculated motion parameters comparison: maximum speed (a), maximum position (b), final position (c) and motion time (d). Comparison of the experimental (blue), and simulated (red), results. The trends are very similar and the simulation captures well platform motion. 


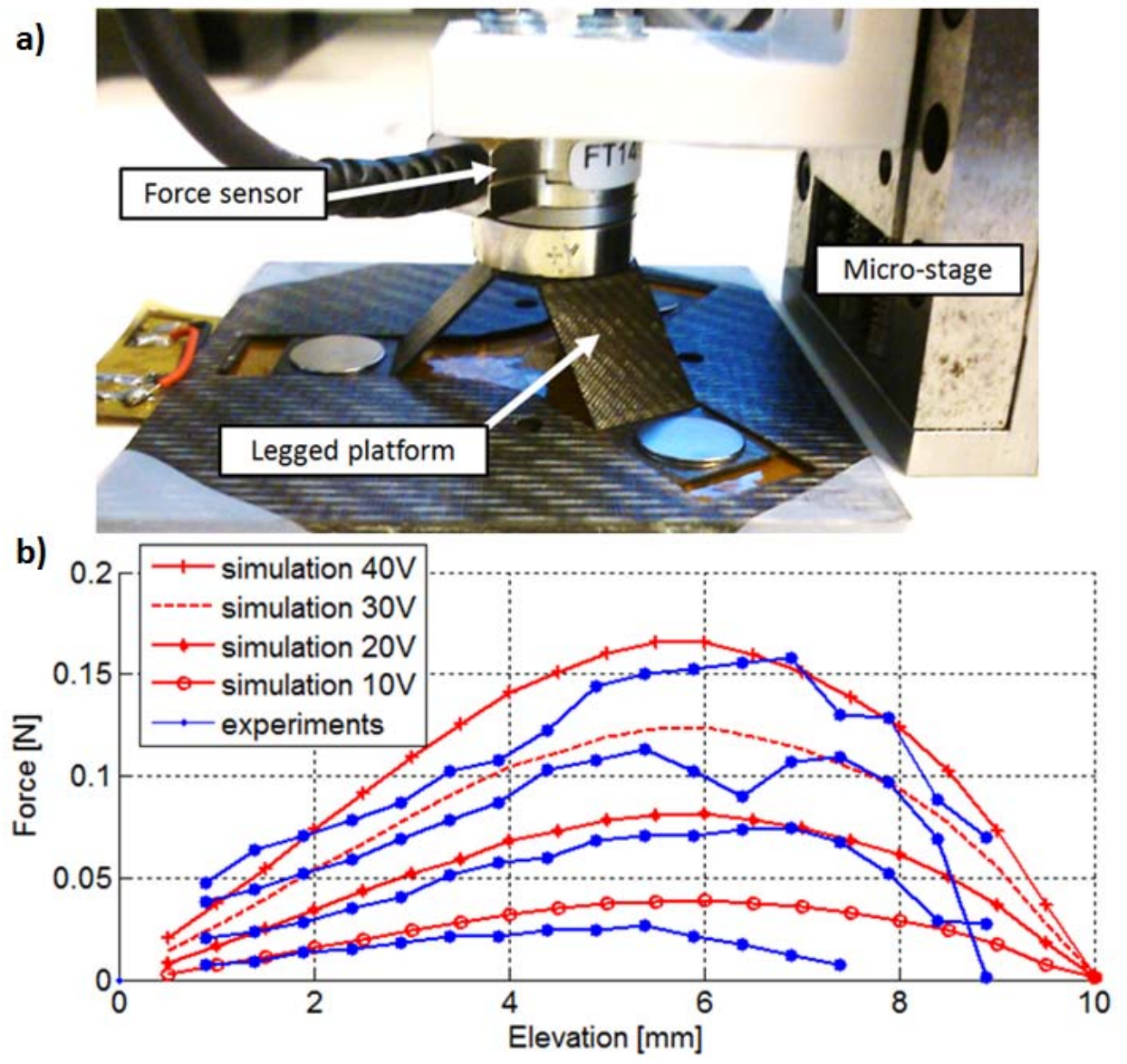

Fig. 21. Legged-system steady forces tests. Experimental setup (a) includes a force sensor and a micro stage placed on top of the platform. The comparison between experimental (blue) and theoretical (red) results is reported (b). 

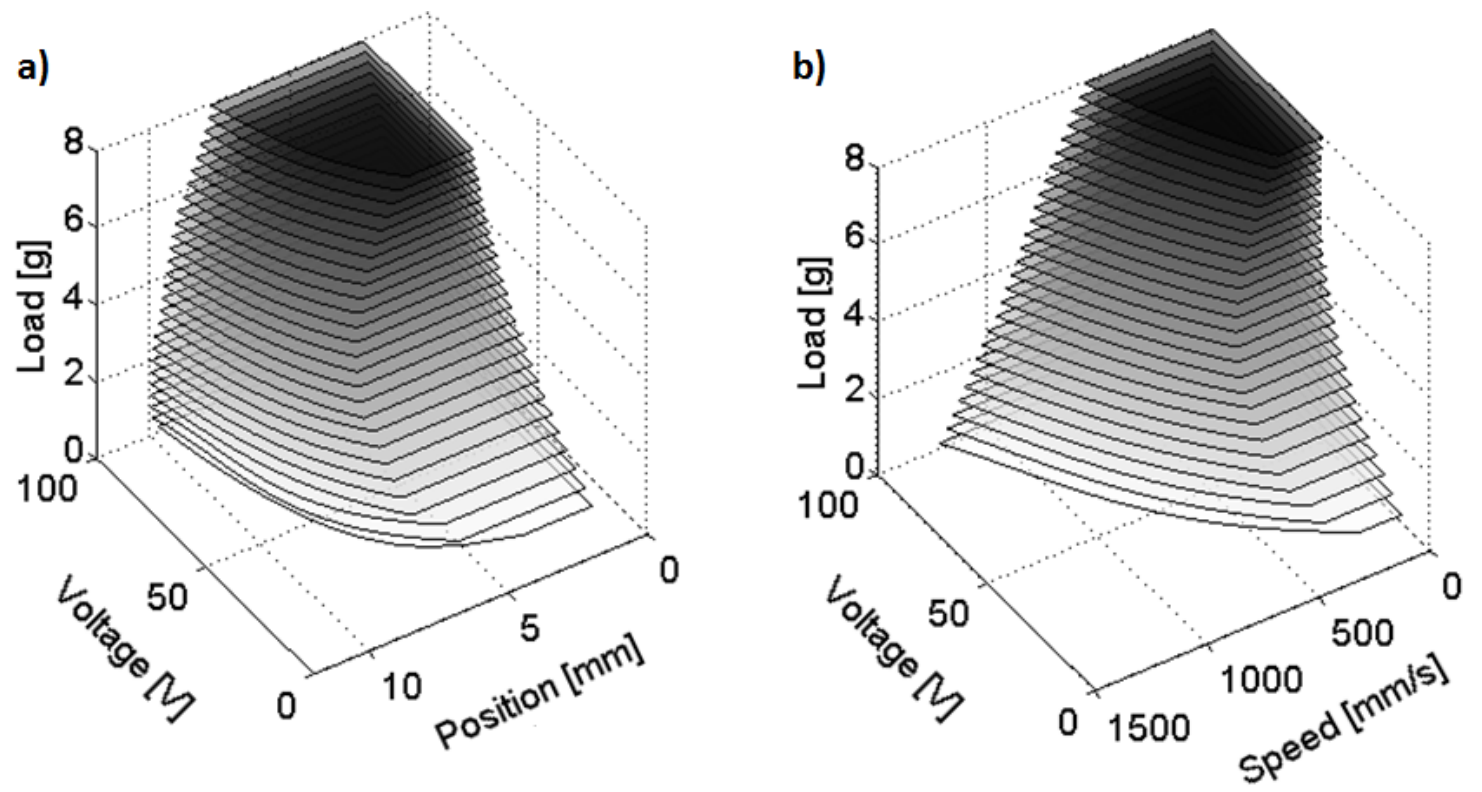

Fig. 22. Pop-up platform with mass on top, simulation results. For different weights and input voltages, the top platform achievable position ranges (a) and speeds (b) are reported. 


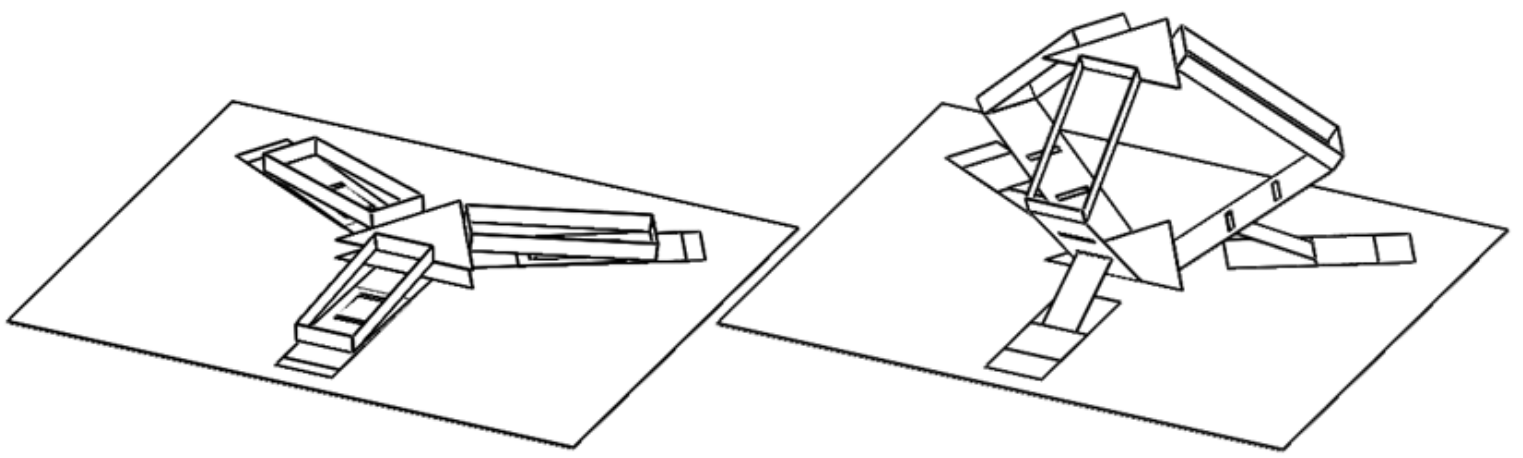

Fig. 23. Envisioned platform where a delta robot is interfaced with the proposed actuation system. Folded portable configuration (left), 3D pop-up configuration (right). 
Tables

TABLE I

USER-DEFINED PARAMETERS USED IN THE FEM SIMULATIONS

\begin{tabular}{ccc}
\hline \hline Parameter Name & $\begin{array}{c}\text { Measur } \\
\text { ement } \\
\text { unit }\end{array}$ & Value \\
\hline Coil inner diameter & $\mathrm{mm}$ & 1 \\
Coil outer diameter & $\mathrm{mm}$ & 12 \\
Coil height & $\mathrm{mm}$ & 0.5 \\
Magnet diameter & $\mathrm{mm}$ & 10 \\
Magnet height & $\mathrm{mm}$ & 1 \\
$\mathrm{Br}$ & $\mathrm{T}$ & 1.2 \\
$\mu_{\mathrm{r}}$ & $/$ & 1.25663 \\
$\sigma$ & $\mathrm{S} / \mathrm{m}$ & $610^{-6}$ \\
$\mathrm{I}_{\mathrm{coil}}$ & $\mathrm{A}$ & 0.23 \\
$\mathrm{~N}$ & $/$ & 300 \\
\hline \hline
\end{tabular}


TABLE II

DESIGN PARAMETERS FOR THE POP-UP PLATFORM

\begin{tabular}{ccc}
\hline \hline $\begin{array}{c}\text { Paramet } \\
\text { er Name }\end{array}$ & $\begin{array}{c}\text { Measure } \\
\text { ment } \\
\text { unit }\end{array}$ & Value \\
\hline $\mathrm{d}$ & $\mathrm{mm}$ & 45 \\
$\mathrm{a}_{\min }$ & $\mathrm{mm}$ & 20 \\
$\mathrm{a}_{\max }$ & $\mathrm{mm}$ & 28 \\
$\mathrm{~b}$ & $\mathrm{~mm}$ & 12 \\
$\mathrm{r}_{\mathrm{p}}$ & $\mathrm{mm}$ & 6 \\
$\mathrm{c}$ & $\mathrm{mm}$ & 16 \\
\hline \hline
\end{tabular}




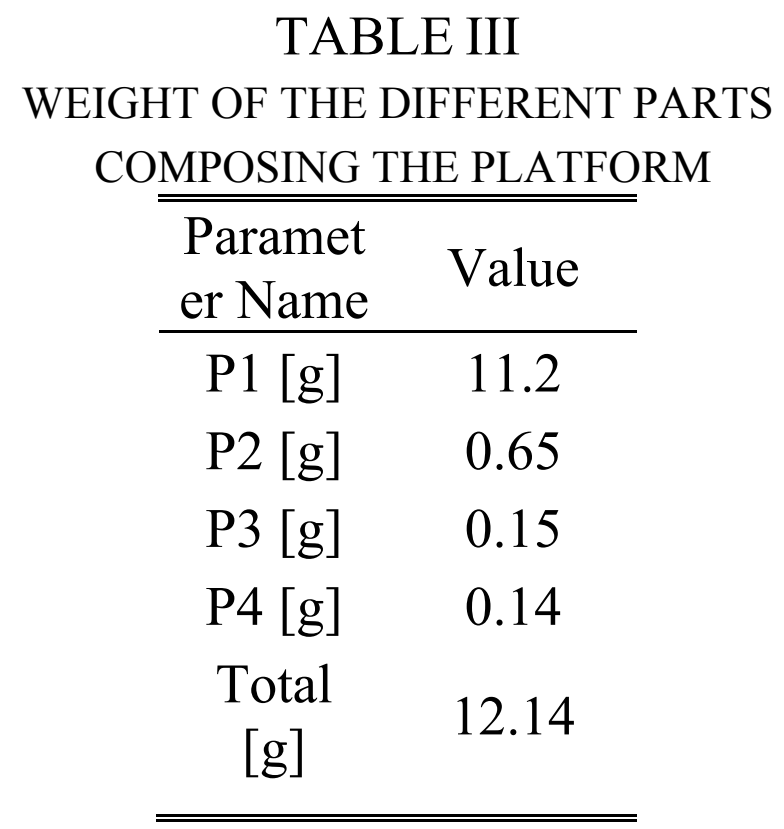


TABLE IV

PARAMETERS USED IN THE THERMAL MODEL

\begin{tabular}{ccc}
\hline \hline $\begin{array}{c}\text { Paramet } \\
\text { er Name }\end{array}$ & $\begin{array}{c}\text { Measur } \\
\text { ement } \\
\text { unit }\end{array}$ & Value \\
\hline $\mathrm{R}_{0}$ & $\Omega$ & 126 \\
$\mathrm{~T}_{0}$ & ${ }^{\circ} \mathrm{C}$ & 20 \\
$\alpha$ & $1 /{ }^{\circ} \mathrm{C}$ & $3.910^{-3}$ \\
$\mathrm{~m}_{\mathrm{c}}$ & $\mathrm{kg}$ & $5.0710^{-5}$ \\
$\mathrm{c}_{\mathrm{c}}$ & $\mathrm{J} /(\mathrm{kg}$ & 385 \\
$\left.{ }^{\circ} \mathrm{C}\right)$ & \\
$\mathrm{m}_{\mathrm{k}}$ & $\mathrm{kg}$ & $1.6810^{-5}$ \\
$\mathrm{c}_{\mathrm{k}}$ & $\mathrm{J} /(\mathrm{kg}$ & 1090 \\
& $\left.{ }^{\circ} \mathrm{C}\right)$ & \\
\hline \hline
\end{tabular}




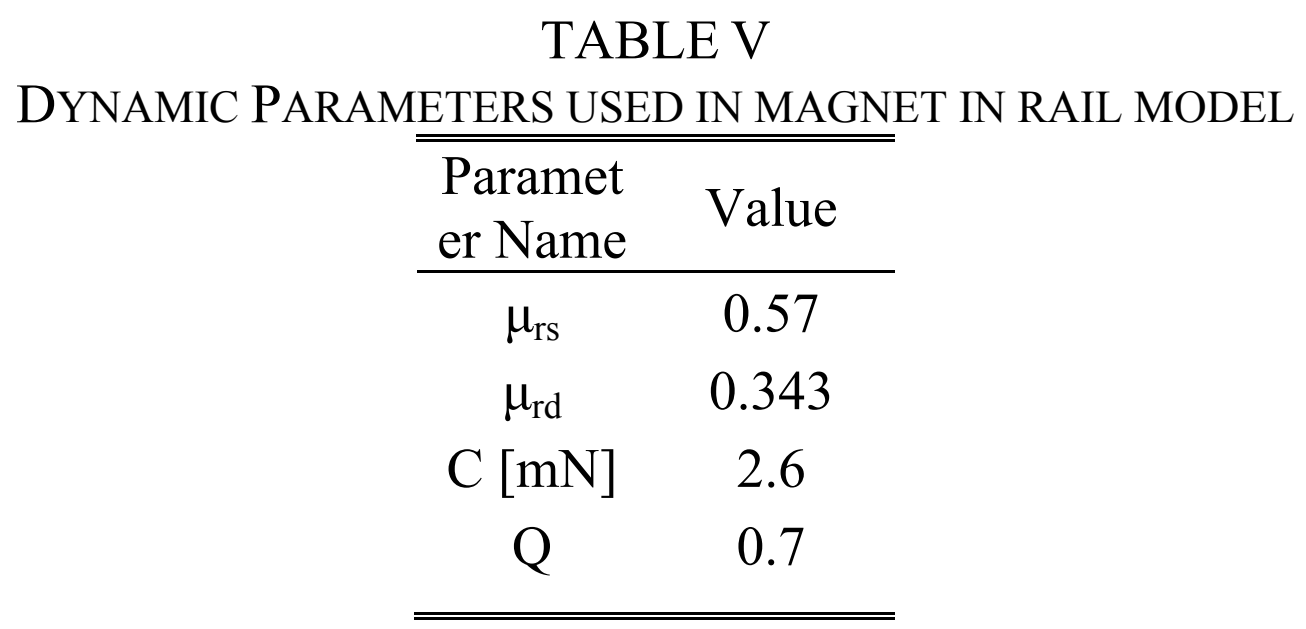

